Pacific

Journal of

Mathematics

MINIMAL SUBMANIFOLDS OF KÄHLER-EINSTEIN MANIFOLDS WITH EQUAL KÄHLER ANGLES

Isabel M.C. Salavessa and Giorgio Valli 


\title{
MINIMAL SUBMANIFOLDS OF KÄHLER-EINSTEIN MANIFOLDS WITH EQUAL KÄHLER ANGLES
}

\author{
Isabel M.C. Salavessa And Giorgio Valli
}

\begin{abstract}
We consider $F: M \rightarrow N$ a minimal submanifold $M$ of real dimension $2 n$, immersed into a Kähler-Einstein manifold $N$ of complex dimension $2 n$, and scalar curvature $R$. We assume that $n \geq 2$ and $F$ has equal Kähler angles. Our main result is to prove that, if $n=2$ and $R \neq 0$, then $F$ is either a complex submanifold or a Lagrangian submanifold. We also prove that, if $n \geq 3, M$ is compact and orientable, then: (A) If $R<0$, then $F$ is complex or Lagrangian; (B) If $R=0$, the Kähler angle must be constant. We also study pluriminimal submanifolds with equal Kähler angles, and prove that, if they are not complex submanifolds, $N$ must be Ricci-flat and there is a natural parallel homothetic isomorphism between $T M$ and the normal bundle.
\end{abstract}

\section{Introduction.}

Let $(N, J, g)$ be a Kähler manifold of complex dimension $2 n$ and $F: M \rightarrow N$ an immersed submanifold of real dimension $2 n$. We denote by $\omega$ the Kähler form of $N, \omega(X, Y)=g(J X, Y)$. On $M$ we take the induced metric $g_{M}=$ $F^{*} g . \quad N$ is Kähler-Einstein if its Ricci tensor is a multiple of the metric, $\operatorname{Ricci}^{N}=R g$. At each point $p \in M$, we identify $F^{*} \omega$ with a skew-symmetric operator of $T_{p} M$ by using the musical isomorphism with respect to $g_{M}$, namely $g_{M}\left(F^{*} \omega(X), Y\right)=F^{*} \omega(X, Y)$. We take its polar decomposition

$$
F^{*} \omega=\widetilde{g} J_{\omega}
$$

where $J_{\omega}: T_{p} M \rightarrow T_{p} M$ is a (in fact unique) partial isometry with the same kernel $\mathcal{K}_{\omega}$ as of $F^{*} w$, and where $\widetilde{g}$ is the positive semidefinite operator $\widetilde{g}=\left|F^{*} \omega\right|=\sqrt{-\left(F^{*} \omega\right)^{2}}$. It turns out that $J_{\omega}: \mathcal{K}_{\omega}^{\perp} \rightarrow \mathcal{K}_{\omega}^{\perp}$ defines a complex structure on $\mathcal{K}_{\omega}^{\perp}$, the orthogonal complement of $\mathcal{K}_{\omega}$ in $T_{p} M$. Moreover, it is $g_{M}$-orthogonal. If we denote by $\Omega_{2 k}^{0}$ the largest open set of $M$ where $F^{*} \omega$ has constant rank $2 k, 0 \leq k \leq n$, then $\mathcal{K}_{\omega}^{\perp}$ is a smooth sub-vector bundle of $T M$ on $\Omega_{2 k}^{0}$. Moreover, $\widetilde{g}$ and $J_{\omega}$ are both smooth on these open sets. The tensor $\widetilde{g}$ is continuous on all $M$ and locally Lipschitz, for the map $P \rightarrow|P|$ is Lipschitz in the space of normal operators. Let $\left\{X_{\alpha}, Y_{\alpha}\right\}_{1 \leq \alpha \leq n}$ 
be a $g_{M}$-orthonormal basis of $T_{p} M$, that diagonalizes $F^{*} \omega$ at $p$, that is

$$
F^{*} \omega=\bigoplus_{0 \leq \alpha \leq n}\left[\begin{array}{cc}
0 & -\cos \theta_{\alpha} \\
\cos \theta_{\alpha} & 0
\end{array}\right],
$$

where $\cos \theta_{1} \geq \cos \theta_{2} \geq \cdots \geq \cos \theta_{n} \geq 0$. The angles $\left\{\theta_{\alpha}\right\}_{1 \leq \alpha \leq n}$ are the Kähler angles of $F$ at $p$. Thus, $\forall \alpha, F^{*} \omega\left(X_{\alpha}\right)=\cos \theta_{\alpha} Y_{\alpha}, F^{*} \omega\left(Y_{\alpha}\right)=$ $-\cos \theta_{\alpha} X_{\alpha}$ and if $k \geq 1$, where $2 k$ is the rank of $F^{*} \omega$ at $p, J_{\omega} X_{\alpha}=Y_{\alpha}$ $\forall \alpha \leq k$. The Weyl's perturbation theorem applied to the eigenvalues of the symmetric operator $\left|F^{*} \omega\right|$ shows that, ordering the $\cos \theta_{\alpha}$ in the above way, the map $p \rightarrow \cos \theta_{\alpha}(p)$ is locally Lipschitz on $M$, for each $\alpha$. A complex direction of $F$ is a real two-plane $P$ of $T_{p} M$ such that $d F(P)$ is a complex line of $T_{F(p)} N$, i.e., $J d F(P) \subset d F(P)$. Similarly, $P$ is said to be a $L a$ grangian direction of $F$ if $\omega$ vanishes on $d F(P)$, that is, $J d F(P) \perp d F(P)$. The immersion $F$ has no complex directions iff $\cos \theta_{\alpha}<1 \forall \alpha$. $\quad M$ is a complex submanifold iff $\cos \theta_{\alpha}=1 \forall \alpha$, and is a Lagrangian submanifold iff $\cos \theta_{\alpha}=0 \forall \alpha$. We say that $F$ has equal Kähler angles if $\theta_{\alpha}=\theta \forall \alpha$. Complex and Lagrangian submanifolds are examples of such case. If $F$ is a complex submanifold, then $J_{\omega}$ is the complex structure induced by $J$ of $N$. The Kähler angles are some functions that at each point $p$ of $M$ measure the deviation of the tangent plane $T_{p} M$ of $M$ from a complex or a Lagrangian subspace of $T_{F(p)} N$. This concept was introduced by Chern and Wolfson $[\mathbf{C h}-\mathbf{W}]$ for oriented surfaces, namely $F^{*} \omega=\cos \theta \mathrm{Vol}_{M}$. This $\cos \theta$ may have negative values and is smooth on all $M$. In our definition, for $n=1$, we demanded $\cos \theta \geq 0$, that is, it is the modulus of the $\cos \theta$ given for surfaces. This may make our $\cos \theta$ do not be smooth. We have chosen this definition, because in higher dimensions we do not have a preferential orientation assigned to the real planes span $\left\{X_{\alpha}, Y_{\alpha}\right\}$.

Our main aim is to find conditions for a minimal submanifold $F$ to be Lagrangian or complex, or $M$ to be a Kähler manifold with respect to $J_{\omega}$. A first result in this direction is due to Wolfson, for the case $n=1$ :

Theorem 1.1 ([W]). If $M$ is a real compact surface and $N$ is a complex Kähler-Einstein surface with $R<0$, anf if $F$ is minimal with no complex points, then $F$ is Lagrangian.

Some results of $[\mathbf{S}-\mathbf{V}]$ are a generalization of the above theorem to higher dimensions. In this paper we study the case of equal Kähler angles. Let us denote by $\nabla_{X} d F(Y)=\nabla d F(X, Y)$ the second fundamental form of $F$. It is a symmetric tensor and takes values in the normal bundle $N M=(d F(T M))^{\perp}$. $F$ is minimal iff trace $_{g_{M}} \nabla d F=0$. Let ( $)^{\perp}$ denote the orthogonal projection of $F^{-1} T N$ onto the normal bundle. If $F$ is an immersion with no complex directions at $p$ and $\left\{X_{\alpha}, Y_{\alpha}\right\}$ diagonalizes $F^{*} \omega$ at $p$, then $\left\{d F\left(Z_{\alpha}\right), d F\left(Z_{\bar{\alpha}}\right)\right.$, $\left.\left(J d F\left(Z_{\alpha}\right)\right)^{\perp},\left(J d F\left(Z_{\bar{\alpha}}\right)\right)^{\perp}\right\}$ constitutes a complex basis of $T_{F(p)}^{c} N$, where 


$$
Z_{\alpha}=\frac{X_{\alpha}-i Y_{\alpha}}{2}=" \alpha ", \quad Z_{\bar{\alpha}}=\overline{Z_{\alpha}}=\frac{X_{\alpha}+i Y_{\alpha}}{2}=" \bar{\alpha} "
$$

are complex vectors of the complexified tangent space of $M$ at $p$. We extend to the complexified vector bundles the Riemannian tensor metric $g_{M}$ (sometimes denoted by $\langle\rangle$,$) , the curvature tensors of M$ and $N$, and any other tensors that will occur, always by $\mathbb{C}$-multilinearity. On $M$ the Ricci tensor of $N$ can be described by the following expression ([S-V]): For $U, V \in T_{F(p)} N$,

$$
\operatorname{Ricci}^{N}(U, V)=\sum_{1 \leq \mu \leq n} \frac{4}{\sin ^{2} \theta_{\mu}} R^{N}\left(U, J V, d F(\mu),(J d F(\bar{\mu}))^{\perp}\right),
$$

where $R^{N}$ denotes the Riemannian curvature tensor of $N$. An application of Codazzi equation to the above expression proves that, if $N$ is KählerEinstein with $R \neq 0$, Theorem 1.1 can be generalized to any dimension for totally geodesic immersions without complex directions ([S-V]).

We can also obtain the same conclusion to "broadly-pluriminimal" immersions for $n=2$, and $N$ Kähler-Einstein with negative Ricci tensor ([S-V]). A minimal immersion $F$ is said to be broadly-pluriminimal, if, for each $p \in \Omega_{2 k}^{0}$, with $k \geq 1, F$ is pluriharmonic with respect to any $g_{M}$-orthogonal complex structure $\widetilde{J}=J_{\omega} \oplus J^{\prime}$ on $T_{p} M$ where $J^{\prime}$ is any $g_{M}$-orthogonal complex structure of $\mathcal{K}_{\omega}$ at $p$, that is, $(\nabla d F)^{(1,1)}=0$. The $(1,1)$-part of $\nabla d F$ is just given by $(\nabla d F)^{(1,1)}(X, Y)=\frac{1}{2}(\nabla d F(X, Y)+\nabla d F(\widetilde{J} X, \widetilde{J} Y)) \forall X, Y \in T_{p} M$. If $\mathcal{K}_{\omega}=0$, this means that $F$ is pluriharmonic with respect to the almost complex structure $J_{\omega}$ (see for example $[\mathbf{O}-\mathbf{V}]$ ). In this case, we say that $F$ is pluriminimal in the usual sense, or simply pluriminimal. Pluriharmonic immersions are obviously minimal. If $F$ has equal Kähler angles, then only $\Omega_{2 n}^{0}$ is considered, where $\mathcal{K}_{\omega}=0$ and $\widetilde{J}=J_{\omega}$. Products of minimal real surfaces of Kähler surfaces, totally geodesic submanifolds, minimal Lagrangian submanifolds, and complex submanifolds are examples of broadly-pluriminimal submanifolds. In Sections 2 and 3, using an isomorphism $\Phi$ from the tangent bundle of $M$ into the normal bundle, we will see that pluriminimal immersions with equal Kähler angles immersed into Kähler-Einstein manifolds, and that are not complex submanifolds, can be interpretated as submanifolds with "torsion free" normal bundle. Moreover, they have constant Kähler angle, and only exist on Ricci-flat manifolds. In this case, $\Phi$ defines a parallel homothetic isomorphism between $T M$ and $N M$.

For a minimal immersion $F$ with no complex directions we consider the locally Lipschitz map, symmetric on the Kähler angles,

$$
\kappa=\sum_{1 \leq \alpha \leq n} \log \left(\frac{1+\cos \theta_{\alpha}}{1-\cos \theta_{\alpha}}\right) .
$$


This map is smooth on each $\Omega_{2 k}^{0}$, nonnegative, and vanishes at Lagrangian points. It is an increasing map on each $\cos \theta_{\alpha}$. In $[\mathbf{S}-\mathbf{V}]$ we have given an expression for $\triangle \kappa$ at a point $p_{0} \in \Omega_{2 k}^{0}$, which we prove in the appendix of this paper, namely,

$$
\begin{aligned}
\triangle \kappa= & 4 i \sum_{\beta} \operatorname{Ricci}^{N}(J d F(\beta), d F(\bar{\beta})) \\
+ & \sum_{\beta, \mu} \frac{32}{\sin ^{2} \theta_{\mu}} \operatorname{Im}\left(R^{N}\left(d F(\beta), d F(\mu), d F(\bar{\beta}), J d F(\bar{\mu})+i \cos \theta_{\mu} d F(\bar{\mu})\right)\right) \\
- & \sum_{\beta, \mu, \rho} \frac{64\left(\cos \theta_{\mu}+\cos \theta_{\rho}\right)}{\sin ^{2} \theta_{\mu} \sin ^{2} \theta_{\rho}} \\
& \cdot \operatorname{Re}\left(g\left(\nabla_{\beta} d F(\mu), J d F(\bar{\rho})\right) g\left(\nabla_{\bar{\beta}} d F(\rho), J d F(\bar{\mu})\right)\right) \\
+ & \sum_{\beta, \mu, \rho} \frac{32\left(\cos \theta_{\rho}-\cos \theta_{\mu}\right)}{\sin ^{2} \theta_{\mu} \sin ^{2} \theta_{\rho}} \\
& \cdot\left(\left|g\left(\nabla_{\beta} d F(\mu), J d F(\rho)\right)\right|^{2}+\left|g\left(\nabla_{\bar{\beta}} d F(\mu), J d F(\rho)\right)\right|^{2}\right) \\
+ & \sum_{\beta, \mu, \rho} \frac{32\left(\cos \theta_{\mu}+\cos \theta_{\rho}\right)}{\sin ^{2} \theta_{\mu}}\left(\left|\left\langle\nabla_{\beta} \mu, \rho\right\rangle\right|^{2}+\left|\left\langle\nabla_{\bar{\beta}} \mu, \rho\right\rangle\right|^{2}\right),
\end{aligned}
$$

where $\left\{X_{\alpha}, Y_{\alpha}\right\}_{1 \leq \alpha \leq n}$ is a $g_{M}$-orthonormal local frame of $M$, with $Y_{\alpha}=$ $J_{\omega} X_{\alpha}$ for $\alpha \leq k,\left\{X_{\alpha}, Y_{\alpha}\right\}_{\alpha \geq k+1}$ any $g_{M}$-orthonormal frame of $\mathcal{K}_{\omega}$, and which at $p_{0}$ diagonalizes $F^{*} \omega$. For $F$ pluriminimal on $\Omega_{2 n}^{0}$ and $N$ KählerEinstein, we can get the following very simple final expression on $\Omega_{2 n}^{0}([\mathbf{S}-\mathbf{V}])$

$$
\triangle \kappa=-2 R\left(\sum_{1 \leq \beta \leq n} \cos \theta_{\beta}\right) .
$$

If $F$ has equal Kähler angles, then the expression of $\triangle \kappa$ given in (1.6) can also be substantially simplified. Minimal surfaces with constant curvature and constant Kähler angle in complex space forms have been classified in [O]. Conditions on the curvature of $M, N$, and/or constant equal Kähler angles lead to some conclusions in our case as well, as we show in the theorems below. Henceforth, we assume $N$ is Kähler-Einstein. The expression for $\triangle \kappa$, where the Ricci tensor of $N$ appears, and the Weitzenböck formula for $F^{*} \omega$, leading to an integral equation involving the scalar curvature $R$, some trigonometric functions of the common Kähler angle, and the gradient of its cosine (Proposition 4.2), are our tools to obtain the results of this paper. In Section 4 we prove our main results, namely: 
Theorem 1.2. Let $F$ be a minimal immersion of a manifold $M$, into a Kähler-Einstein manifold $N$, with equal Kähler angles.

(i) If $n=2$ and $R \neq 0$, then $F$ is either a complex or a Lagrangian submanifold.

(ii) If $n \geq 3, M$ is compact, orientable, $R<0$, then $F$ is either a complex or Lagrangian submanifold.

(iii) If $n \geq 3, M$ is compact, orientable, $R=0$, then the common Kähler angle must be constant.

The conclusions in (i) and (ii) give a generalization of Theorem 1.1 to higher dimensions and equal Kähler angles. The case $n=2$ is the most special, because, in this dimension, immersions with equal Kähler angles have harmonic $F^{*} \omega$, as we will see in Section 3. The cases $n=3$ or 4 also have special properties. If the angle is constant we may allow $R>0$ :

Theorem 1.3. Let $F$ be minimal with constant equal Kähler angles, $M$ compact, orientable, and $R \neq 0$. Then, $F$ is either a complex or a Lagrangian submanifold.

Theorem 1.4. Let $F$ be minimal with equal Kähler angles, and $M$ compact, orientable, with nonnegative isotropic scalar curvature. If $n=2,3$ or 4 , then one of the following cases holds:

(i) $M$ is a complex submanifold of $N$.

(ii) $M$ is a Lagrangian submanifold of $N$.

(iii) $R=0$ and $\cos \theta=$ constant $\neq 0,1, J_{\omega}$ is a complex integrable structure, with $\left(M, J_{\omega}, g_{M}\right)$ a Kähler manifold.

For any $n \geq 1$, any $R$, and constant equal Kähler angle, (i), (ii) or (iii) hold as well.

This theorem can be applied, for instance, to flat minimal tori on CalabiYau manifolds, or to spheres or products of $S^{2}$ with $S^{2}$ or with flat tori minimaly immersed into Kähler-Einstein manifolds with positive scalar curvature.

\section{The morphism $\Phi$.}

We consider the following morphism of vector bundles

$$
\begin{array}{rlc}
\Phi: T M & \rightarrow & N M \\
X & \rightarrow & (J d F(X))^{\perp} .
\end{array}
$$

We easily verify that

$$
\Phi(X)=J d F(X)-d F\left(F^{*} \omega(X)\right) .
$$

Both $T M$ and $N M$ are real vector bundles of the same dimension $2 n . F$ has no complex directions iff $\Phi$ is an isomorphism. In fact $\Phi(X)=0$, 
iff $J d F(X)=d F(Y)$ for some $Y$, i.e., $\operatorname{span}\{X, Y=$ " $J X "\}$ is a complex direction of $F$. Assume there are no complex directions. Then,

$$
\hat{g}(X, Y)=g_{M}(X, Y)-g_{M}\left(F^{*} \omega(X), F^{*} \omega(Y)\right)
$$

defines a Riemannian metric on $M$. With this metric, $\Phi:(T M, \hat{g}) \rightarrow$ $(N M, g)$ is an isomorphism of Riemannian vector bundles. Let us denote by $\nabla, \hat{\nabla}, \nabla^{\perp}$, and $\nabla^{\prime}$, respectively, the Levi-Civita connection of $\left(M, g_{M}\right)$, the Levi-Civita connection of $(M, \hat{g})$, the usual connection of $N M$ induced by the Levi-Civita connection of $N$, and the connection on $T M$ that makes the isomorphism $\Phi$ parallel, namely $\nabla^{\prime}=\Phi^{-1 *} \nabla^{\perp}$. We will also denote by $\nabla$ the Levi-Civita connection of $N$ and the induced connection on $F^{-1} T N$, as well. Thus, if $U$ is a smooth section of $N M \subset F^{-1} T N$, and $X, Y$ are smooth vector fields on $M$, we have

$$
\nabla_{X}^{\perp} U=\left(\nabla_{X} U\right)^{\perp} \quad \Phi\left(\nabla_{X}^{\prime} Y\right)=\nabla_{X}^{\perp}(\Phi(Y)) .
$$

The connections $\nabla$ and $\hat{\nabla}$ have no torsion, because they are Levi-Civita, but $\nabla^{\prime}$ may have nonzero torsion $T^{\prime}$. Since both $\hat{\nabla}$ and $\nabla^{\prime}$ are Riemannian connections of $T M$ for the same Riemannian metric $\hat{g}$, then $T^{\prime}=0$ iff $\hat{\nabla}=\nabla^{\prime}$ iff $\Phi$ is parallel. Note that, if $F$ is Lagrangian, then $\Phi(X)=$ $J d F(X) \in N M, J(N M)=d F(T M)$, and $\hat{g}=g_{M}, \hat{\nabla}=\nabla$. Therefore, $\nabla_{X} \Phi(Y)=\left(J \nabla_{X} d F(Y)\right)^{\perp}=0$, that is, $\Phi$ is parallel, and so $\nabla^{\prime}=\nabla$, as well. In the next section (Corollary 3.2), we will see a converse of this. We extend $\Phi: T M^{c} \rightarrow N M^{c}$ to the complexified spaces by $\mathbb{C}$-linearity.

Lemma 2.1. If $\left\{X_{\alpha}, Y_{\alpha}\right\}$ is a diagonalizing $g_{M}$-orthonormal basis of $F^{*} \omega$ at $p$, then at $p$, and for each $\alpha, \beta$

$$
\begin{aligned}
& \Phi\left(T^{\prime}\left(Z_{\alpha}, Z_{\bar{\beta}}\right)\right)=i\left(\cos \theta_{\alpha}+\cos \theta_{\beta}\right) \nabla_{Z_{\alpha}} d F\left(Z_{\bar{\beta}}\right) \\
& \Phi\left(T^{\prime}\left(Z_{\alpha}, Z_{\beta}\right)\right)=i\left(\cos \theta_{\alpha}-\cos \theta_{\beta}\right) \nabla_{Z_{\alpha}} d F\left(Z_{\beta}\right) .
\end{aligned}
$$

Proof.

$$
\begin{aligned}
\Phi\left(\nabla_{X}^{\prime} Y\right) & =\nabla_{X}^{\perp}(\Phi(Y))=\left(\nabla_{X}(\Phi(Y))\right)^{\perp} \\
& =\left(\nabla_{X}\left(J d F(Y)-d F\left(F^{*} \omega(Y)\right)\right)\right)^{\perp} \\
& =\left(J \nabla_{X} d F(Y)+J d F\left(\nabla_{X} Y\right)-\nabla_{X} d F\left(F^{*} \omega(Y)\right)\right)^{\perp} .
\end{aligned}
$$

Therefore, using the symmetry of the $\nabla d F$ and the fact that $\nabla$ is torsionless,

$$
\begin{aligned}
\Phi\left(T^{\prime}(X, Y)\right) & =\Phi\left(\nabla_{X}^{\prime} Y-\nabla_{Y}^{\prime} X-[X, Y]\right) \\
& =-\nabla_{X} d F\left(F^{*} \omega(Y)\right)+\nabla_{Y} d F\left(F^{*} \omega(X)\right) .
\end{aligned}
$$

The lemma follows now immediately. 
For each $U \in N M_{p}$, let us denote by $A^{U}: T_{p} M \rightarrow T_{p} M$ the symmetric operator $g_{M}\left(A^{U}(X), Y\right)=g(\nabla d F(X, Y), U)$. From Lemma 2.1 and (2.3) we have:

Proposition 2.1. If $F$ is an immersion without complex directions, then:

(i) $\Phi$ is parallel iff $F^{*} \omega$ anti-commutes with $A^{U}, \forall U \in N M$.

(ii) If $F$ has equal Kähler angles, on $\Omega_{2 n}^{0}, T^{\prime}$ is of type $(1,1)$ with respect to $J_{\omega}$.

(iii) On $\Omega_{2 n}^{0}, F$ is pluriminimal iff $T^{\prime}$ is of type $(2,0)+(0,2)$ with respect to $J_{\omega}$.

Remark 1. If we call $\omega_{N M}$ the restriction of the Kähler form $\omega$ to the normal bundle $N M$, we see that, if $\left\{X_{\alpha}, Y_{\alpha}\right\}$ is a diagonalizing $g_{M}$-orthonormal basis of $F^{*} \omega$ at a point $p$, then $\left\{U_{\alpha}=\Phi\left(\frac{Y_{\alpha}}{\sin \theta_{\alpha}}\right), V_{\alpha}=\Phi\left(\frac{X_{\alpha}}{\sin \theta_{\alpha}}\right)\right\}$ is a diagonalizing $g$-orthonormal basis of $\omega_{N M}$. Moreover, $N M$ has the same Kähler angles as $F$. Let $J_{N M}$ denote the complex structure on $N M$ defined by this basis, that is, the one that comes from the polar decomposition of $\omega_{N M}$. Then, $\Phi J_{\omega}=-J_{N M} \Phi$.

We should also remark the following:

Proposition 2.2. If $F$ is an immersion with parallel 2-form $F^{*} \omega$, then the Kähler angles are constant and, in particular, $M=\Omega_{2 k}^{0}$ for some $k$. In this case, considering TM with the Levi-Civita connection $\nabla, \mathcal{K}_{\omega}$ and $\mathcal{K}_{\omega}^{\perp}$ are parallel sub-vector bundles of $T M$, and $J_{\omega} \in C^{\infty}\left(\mathcal{K}_{\omega}^{\perp *} \otimes \mathcal{K}_{\omega}^{\perp}\right)$, $\widetilde{g}, \hat{g} \in C^{\infty}\left(\bigodot^{2} T^{*} M\right)$ are parallel sections. Furthermore, $(X, Y, Z) \rightarrow$ $g\left(\nabla_{Z} d F(X), J d F(Y)\right)$ is symmetric on $T M$, and, if $F$ has no complex directions, $\hat{\nabla}=\nabla$. Moreover, if $\cos \theta_{\alpha_{1}}>\cdots>\cos \theta_{\alpha_{r}}$ are the distinct eigenvalues of $F^{*} \omega$, the corresponding eigenspaces $E_{\alpha_{t}}$ define a smooth integrable distribution of TM whose integral submanifolds are totally geodesic submanifolds of $M$. The integral submanifolds of $E_{\alpha_{r}}$ are isotropic in $N$ if $\cos \theta_{\alpha_{r}}=0$, and the ones of $E_{\alpha_{1}}$ are complex submanifolds of $N$ if $\cos \theta_{\alpha_{1}}=1$. The other ones are Kähler manifolds with respect to $J_{\omega}$, and $F$ restricted to each one of them is an immersion of constant equal Kähler angles $\theta_{\alpha_{t}}$ with respect to $J$.

Proof. If $X, Y$ are smooth vector fields on $M$ and $Z \in T_{p} M$, an elementary computation gives

$$
\nabla_{Z} F^{*} \omega(X, Y)=-g\left(\nabla_{Z} d F(X), J d F(Y)\right)+g\left(\nabla_{Z} d F(Y), J d F(X)\right),
$$

which proves the symmetry of $(X, Y, Z) \rightarrow g\left(\nabla_{Z} d F(X), J d F(Y)\right)$. From (2.2) we see that $\hat{g}$ is parallel. Consequently, away from complex directions, $\nabla=\hat{\nabla}$. If we parallel transport a diagonalizing orthonormal basis $\left\{X_{\alpha}, Y_{\alpha}\right\}$ of $F^{*} \omega$ at $p_{0}$ along geodesics, on a neighbourhood of $p_{0}$, since $F^{*} \omega$ is parallel 
we get a diagonalizing orthonormal frame on a whole neighbourhood with the property $\nabla X_{\alpha}\left(p_{0}\right)=\nabla Y_{\alpha}\left(p_{0}\right)=0$. It also follows that $\cos \theta_{\alpha}$ remains constant along geodesics, so it is constant, and $J_{\omega}\left(X_{\alpha}\right)=Y_{\alpha}$ on a neighbourhood of $p_{0}$, with $\nabla J_{\omega}=0$ at $p_{0}$, and so $J_{\omega}$ is parallel. Similarly we see that $\widetilde{g}$ is parallel. If we extend $F^{*} \omega$ to the complexified tangent space $T_{p_{0}}^{c} M$, then $F^{*} \omega\left(Z_{\alpha}\right)=i \cos \theta_{\alpha} Z_{\alpha}$, and $F^{*} \omega\left(Z_{\bar{\alpha}}\right)=-i \cos \theta_{\alpha} Z_{\bar{\alpha}}$. Obviously, the corresponding eigenspaces of $F^{*} \omega$, are parallel sub-vector bundles of $T^{c} M$.

\section{Immersions with equal Kähler angles.}

If $F$ has equal Kähler angles, then

$$
F^{*} \omega=\cos \theta J_{\omega} \text { and } \hat{g}=\sin ^{2} \theta g_{M},
$$

with $\cos \theta$ a locally Lipschitz map on $M$, smooth on the open set where it does not vanish, and $\Omega_{2 k}^{0}=\emptyset \forall k \neq 0, n$. Note that $\sin ^{2} \theta$ and $\cos ^{2} \theta$ are smooth on all $M$. The set $\mathcal{L}=\cos \theta^{-1}(\{0\})$ is the set of Lagrangian points, for, at these points, the tangent space of $M$ is a Lagrangian subspace of the tangent space of $N$. Similarly, we say that $\mathcal{C}=\cos \theta^{-1}(\{1\})$ is the set of complex points. If $M$ has a fixed orientation, we can distinguish the set of well-oriented complex points from the twisted complex points, according $J_{\omega}$ defines the same or the opposite orientation. On the open set $\Omega_{2 n}^{0}=\cos \theta^{-1}(\mathbb{R} \sim\{0\})=M \sim \mathcal{L}, J_{\omega}$ defines a smooth almost complex structure $g_{M}$-orthogonal. On the open set $\cos \theta^{-1}(\mathbb{R} \sim\{1\})=M \sim \mathcal{C}, \hat{g}$ is a smooth metric conformally equivalent to $g_{M}$. Thus, if $n \geq 2, \hat{\nabla}=\nabla$ iff $\theta$ is constant. Since the Kähler angles are equal, any smooth local orthonormal frame of the type $\left\{X_{\alpha}, Y_{\alpha}=J_{\omega} X_{\alpha}\right\}$ diagonalizes $F^{*} \omega$ on the whole set where it is defined. Differentiating $F^{*} \omega=\cos \theta J_{\omega}$, we get $\nabla_{X} F^{*} \omega=d \cos \theta(X) J_{\omega}+$ $\cos \theta \nabla_{X} J_{\omega}$, with $J_{\omega}$ orthogonal to $\nabla_{X} J_{\omega}$ with respect to the Hilbert-Schmidt inner product (because $\left\|J_{\omega}\right\|^{2}=2 n$ is constant). Hence, considering $F^{*} \omega$ an operator on $T M$, on $\Omega_{2 n}^{0} \cup \Omega_{0}^{0}$

$$
\left\|\nabla F^{*} \omega\right\|^{2}=2 n\|\nabla \cos \theta\|^{2}+\cos ^{2} \theta\left\|\nabla J_{\omega}\right\|^{2} .
$$

Then, on $\Omega_{2 n}^{0}, \nabla F^{*} \omega=0$ iff $\nabla J_{\omega}=0$ and $\theta$ is constant. Note that $\left\|\nabla F^{*} \omega\right\|^{2}$, considering $F^{*} \omega$ an operator on $T M$, is twice the square norm when considering $F^{*} \omega$ a 2-form. From (2.3) we get, on $M \sim \mathcal{C}$,

$$
\Phi\left(T^{\prime}(X, Y)\right)=2 \cos \theta(\nabla d F)^{(1,1)}\left(J_{\omega} X, Y\right) .
$$

The right-hand side of (3.2) is defined to be zero at a Lagrangian point. Consequentely

Proposition 3.1. If $F$ is an immersion with equal Kähler angles and without complex points, then $T^{\prime}=0$, that is, $\nabla^{\prime}=\hat{\nabla}$ iff $\Phi$ is parallel iff $F$ is 
Lagrangian or pluriminimal. In particular, if $F$ is minimal, $\Phi$ is parallel iff $F$ is broadly-pluriminimal.

This also holds for $n=1$, where pluriminimality condition coincides with minimality. In this case $\pm J_{\omega}$ is the natural (local) complex structure of the surface (the sign depends on the (local) chosen orientation). Let $\operatorname{Re}(u+i v)=$ $u$, for $u, v \in N M$.

Proposition 3.2. If $F$ is any immersion with equal Kähler angles, then, away from complex and Lagrangian points,

$$
\begin{aligned}
& \Phi\left(\frac{1-n}{4} \nabla \log \sin ^{2} \theta\right) \\
& =\frac{4 \cos \theta}{\sin ^{2} \theta} \operatorname{Re}\left(i \sum_{\beta, \mu}\left(g\left(\nabla_{\bar{\mu}} d F(\mu), J d F(\beta)\right)-g\left(\nabla_{\bar{\mu}} d F(\beta), J d F(\mu)\right)\right) \Phi(\bar{\beta})\right),
\end{aligned}
$$

where $\nabla \log \sin ^{2} \theta$ is the gradient with respect to $g_{M}$.

If $F$ is a complex submanifold on a open set, then $J_{\omega}$ is the induced complex structure on $M$ and $\nabla d F$ is of type $(2,0)$. Applying Proposition 2.2 on $\Omega_{0}^{0}$, and Proposition 3.2 on open sets without complex and Lagrangian points, and noting that $\{\Phi(\beta), \Phi(\bar{\beta})=\overline{\Phi(\beta)}\}_{1 \leq \beta \leq n}$ multiplied by $\frac{\sqrt{2}}{\sin \theta}$ constitutes an unitary basis of $N M^{c}$, we immediately conclude:

Corollary 3.1. If $F$ is an immersion with equal Kähler angles, and $n \geq 2$, then $\theta$ is constant iff

$$
\sum_{\mu} g\left(\nabla_{\bar{\mu}} d F(\mu), J d F(\beta)\right)=\sum_{\mu} g\left(\nabla_{\bar{\mu}} d F(\beta), J d F(\mu)\right) \quad \forall \beta .
$$

Note that (3.3) is a sort of symmetry property, and the first term is just $\frac{n}{2} g(H, J d F(\beta))$, where $H=\frac{1}{2 n} \operatorname{trace}_{g_{M}} \nabla d F=\frac{2}{n} \sum_{\mu} \nabla d F(\bar{\mu}, \mu)$ is the mean curvature of $F$.

Theorem 3.1. If $n \geq 2$ and $F$ is a pluriminimal immersion with equal Kähler angles then $\cos \theta=$ constant. Moreover, if it is not a complex submanifold, then $\nabla=\hat{\nabla}=\nabla^{\prime}$, and $N$ must be Ricci-flat. In particular, $\Phi$ defines a parallel homothetic isomorphism from $\left(T M, g_{M}\right)$ onto $(N M, g)$.

Proof. On a neighbourhood of a non-complex point, from Proposition 3.1, $\hat{\nabla}=\nabla^{\prime}$, and from Corollary 3.1, $\cos \theta$ is constant. Then $\hat{\nabla}=\nabla$, as well. So if $F$ is not a complex submanifold, it has no complex points anywhere. Finally, (1.7) for pluriminimal immersions with $\kappa=$ constant gives $R=0$.

The above theorem and Proposition 3.1 lead to:

Corollary 3.2. If $F$ is a minimal immersion with equal Kähler angles, without complex points, $n \geq 2$, and $R \neq 0$, then $F$ is Lagrangian iff $\Phi$ is parallel. 
To prove Proposition 3.2 we will need to relate the three connections of $M, \nabla, \hat{\nabla}$ and $\nabla^{\prime}$. Let $\left\{e_{1}, \ldots, e_{2 n}\right\}=\left\{X_{\mu}, Y_{\mu}=J_{\omega} X_{\mu}\right\}_{1 \leq \mu \leq n}$ be a local $g_{M}$-orthonormal frame away from the Lagrangian and complex set, and $\partial_{1}, \ldots, \partial_{2 n}$ a local frame of $M$ defined by a coordinate chart. Set $g_{i j}=$ $g_{M}\left(\partial_{i}, \partial_{j}\right), \hat{g}_{i j}=\hat{g}\left(\partial_{i}, \partial_{j}\right)=\sin ^{2} \theta g_{i j}$, and $e_{s}=\sum_{i} \lambda_{s i} \partial_{i}$. The Christofel symbols are given by $2 \hat{\Gamma}_{i j}^{k}=\sum_{s} \hat{g}^{k s}\left(\partial_{i} \hat{g}_{s j}+\partial_{j} \hat{g}_{i s}-\partial_{s} \hat{g}_{i j}\right)=\delta_{k j} \partial_{i} \log \sin ^{2} \theta+$ $\delta_{k i} \partial_{j} \log \sin ^{2} \theta-\sum_{s} g^{k s} g_{i j} \partial_{s} \log \sin ^{2} \theta+2 \Gamma_{i j}^{k}$. Hence

$$
\begin{aligned}
\hat{\nabla}_{\partial_{i}} \partial_{j}-\nabla_{\partial_{i}} \partial_{j} & =\sum_{k}\left(\hat{\Gamma}_{i j}^{k}-\Gamma_{i j}^{k}\right) \partial_{k} \\
& =\frac{1}{2}\left(\partial_{i}\left(\log \sin ^{2} \theta\right) \partial_{j}+\partial_{j}\left(\log \sin ^{2} \theta\right) \partial_{i}-g_{i j} \nabla\left(\log \sin ^{2} \theta\right)\right) .
\end{aligned}
$$

Since $\sum_{i j} g_{i j} \lambda_{s i} \lambda_{s j}=1, \sum_{s} \hat{\nabla}_{e_{s}} e_{s}-\nabla_{e_{s}} e_{s}=\sum_{s i j} \lambda_{s i} \lambda_{s j}\left(\hat{\nabla}_{\partial_{i}} \partial_{j}-\nabla_{\partial_{i}} \partial_{j}\right)=$ $(1-n) \nabla \log \sin ^{2} \theta$. Therefore,

$$
\begin{aligned}
\sum_{\mu} \hat{\nabla}_{\bar{\mu}} \mu-\nabla_{\bar{\mu}} \mu= & \frac{1}{4} \sum_{\mu}\left(\hat{\nabla}_{X_{\mu}} X_{\mu}+\hat{\nabla}_{Y_{\mu}} Y_{\mu}-\nabla_{X_{\mu}} X_{\mu}-\nabla_{Y_{\mu}} Y_{\mu}\right) \\
& -i\left(\hat{\nabla}_{X_{\mu}} Y_{\mu}-\hat{\nabla}_{Y_{\mu}} X_{\mu}-\nabla_{X_{\mu}} Y_{\mu}+\nabla_{Y_{\mu}} X_{\mu}\right) \\
= & \frac{1}{4} \sum_{s}\left(\hat{\nabla}_{e_{s}} e_{s}-\nabla_{e_{s}} e_{s}\right)+\frac{i}{4} \sum_{\mu}\left(\left[Y_{\mu}, X_{\mu}\right]-\left[Y_{\mu}, X_{\mu}\right]\right) \\
= & \frac{(1-n)}{4} \nabla \log \sin ^{2} \theta .
\end{aligned}
$$

Set $S^{\prime}(X, Y)=\nabla_{X}^{\prime} Y-\hat{\nabla}_{X} Y$. Then $S^{\prime}(X, Y)-S^{\prime}(Y, X)=T^{\prime}(X, Y)$. Similarly we get

$$
\sum_{\mu} \nabla_{\bar{\mu}}^{\prime} \mu-\hat{\nabla}_{\bar{\mu}} \mu=\frac{1}{4} \operatorname{trace}_{g_{M}} S^{\prime}-\frac{i}{4} \sum_{\mu} T^{\prime}\left(X_{\mu}, Y_{\mu}\right) .
$$

Lemma 3.1. $\forall X \in T_{p} M, \quad \sum_{i} \hat{g}\left(S^{\prime}\left(e_{i}, e_{i}\right), X\right)=-\sum_{i} \hat{g}\left(T^{\prime}\left(e_{i}, X\right), e_{i}\right)$.

Proof. We may assume that the local referencial $\partial_{i}$ is $\hat{g}$-orthonormal at a fixed point $p_{0}$. On a neighbourhood of $p_{0}$, we define $\Gamma_{i j}^{\prime k}$ and ${S^{\prime}}_{i j}^{k}$ as

$$
\nabla_{\partial_{i}}^{\prime} \partial_{j}=\sum_{k} \Gamma_{i j}^{\prime k} \partial_{k}, \quad S^{\prime}\left(\partial_{i}, \partial_{j}\right)=\sum_{k} S_{i j}^{\prime k} \partial_{k}=\sum_{k}\left(\Gamma^{\prime k}{ }_{i j}-\hat{\Gamma}_{i j}^{k}\right) \partial_{k} .
$$

Then $T_{i j}^{\prime k}=\Gamma_{i j}^{\prime k}-\Gamma_{j i}^{\prime k}$, and, at $p_{0}, \Gamma^{\prime k}{ }_{i j}=\hat{g}\left(\nabla_{\partial_{i}}^{\prime} \partial_{j}, \partial_{k}\right),{S^{\prime}}_{i j}^{k}=\hat{g}\left(S^{\prime}\left(\partial_{i}, \partial_{j}\right), \partial_{k}\right)$ $=\Gamma_{i j}^{\prime k}-\hat{\Gamma}_{i j}^{k}$. $\nabla^{\prime}$ is a Riemannian connection with respect to $\hat{g}$. Then

$$
\partial_{i} \hat{g}_{j k}\left(p_{0}\right)=\hat{g}\left(\nabla_{\partial_{i}}^{\prime} \partial_{j}, \partial_{k}\right)+\hat{g}\left(\partial_{j}, \nabla_{\partial_{i}}^{\prime} \partial_{k}\right)=\Gamma_{i j}^{\prime k}+\Gamma_{i k}^{\prime j} .
$$


Hence, at $p_{0}$

$$
\begin{aligned}
2 \hat{\Gamma}_{i j}^{k} & =\sum_{s} \hat{g}^{k s}\left(\partial_{i} \hat{g}_{s j}+\partial_{j} \hat{g}_{i s}-\partial_{s} \hat{g}_{i j}\right) \\
& =\Gamma_{i k}^{\prime j}+\Gamma_{i j}^{\prime k}+\Gamma_{j i}^{\prime k}+\Gamma_{j k}^{\prime i}-\Gamma_{k i}^{\prime j}-\Gamma_{k j}^{\prime i} \\
& =\left(\Gamma^{\prime k}{ }_{i j}+\Gamma^{\prime k}{ }_{j i}\right)+\left(\Gamma_{i k}^{\prime j}-\Gamma_{k i}^{\prime j}\right)+\left(\Gamma_{j k}^{\prime i}-\Gamma_{k j}^{\prime i}\right) \\
& =\left(\Gamma^{\prime k}{ }_{i j}+\Gamma_{j i}^{\prime k}\right)+T_{i k}^{\prime j}+T_{j k}^{\prime i} .
\end{aligned}
$$

But $\Gamma_{i j}^{\prime k}+\Gamma^{\prime k}{ }_{j i}=2 \Gamma_{i j}^{\prime k}+\left({\Gamma^{\prime}}_{j i}^{k}-\Gamma^{\prime k}{ }_{i j}\right)=2 \Gamma^{\prime k}{ }_{i j}+T^{\prime k}{ }_{j i}$. Thus

$$
S_{i j}^{\prime k}=\Gamma_{i j}^{\prime k}-\hat{\Gamma}_{i j}^{k}=\frac{1}{2}\left(T_{i j}^{\prime k}-T_{i k}^{\prime j}+T_{k j}^{\prime i}\right)
$$

That is, at $p_{0}, \hat{g}\left(S^{\prime}\left(\partial_{i}, \partial_{j}\right), \partial_{k}\right)=\frac{1}{2}\left(\hat{g}\left(T^{\prime}\left(\partial_{i}, \partial_{j}\right), \partial_{k}\right)-\hat{g}\left(T^{\prime}\left(\partial_{i}, \partial_{k}\right), \partial_{j}\right)+\right.$ $\left.\hat{g}\left(T^{\prime}\left(\partial_{k}, \partial_{j}\right), \partial_{i}\right)\right)$. We may assume that, at $p_{0}, \partial_{i}\left(p_{0}\right)=\frac{e_{i}}{\sin \theta}$, leading to the Lemma.

Proof of Proposition 3.2. Following the proof of Lemma 2.1, $\Phi\left(\nabla_{X}^{\prime} \mu-\nabla_{X} \mu\right)$ $=\left((J-i \cos \theta) \nabla_{X} d F(\mu)\right)^{\perp}$. Hence, from (3.4),

$$
\begin{aligned}
\Phi\left(\frac{(1-n)}{4} \nabla \log \sin ^{2} \theta\right) & =\Phi\left(\sum_{\mu} \hat{\nabla}_{\bar{\mu}} \mu-\nabla_{\bar{\mu}} \mu\right) \\
& =\left((J-i \cos \theta) \frac{n H}{2}\right)^{\perp}-\sum_{\mu} \Phi\left(\nabla_{\bar{\mu}}^{\prime} \mu-\hat{\nabla}_{\bar{\mu}} \mu\right) .
\end{aligned}
$$

But, from (3.5), $\sum_{\mu} \Phi\left(\nabla_{\bar{\mu}}^{\prime} \mu-\hat{\nabla}_{\bar{\mu}} \mu\right)=\frac{1}{4} \Phi\left(\operatorname{trace}_{g_{M}} S^{\prime}\right)-\frac{i}{4} \Phi\left(\sum_{\mu} T^{\prime}\left(X_{\mu}, Y_{\mu}\right)\right)$. The skew-symmetry of $T^{\prime}$ and (3.2) implies that

$$
\Phi\left(\sum_{\mu} T^{\prime}\left(X_{\mu}, Y_{\mu}\right)\right)=-2 i \sum_{\mu} \Phi\left(T^{\prime}(\mu, \bar{\mu})\right)=4 \cos \theta \nabla_{\mu} d F(\bar{\mu})=2 n \cos \theta H
$$

Thus, $\sum_{\mu} \Phi\left(\nabla_{\bar{\mu}}^{\prime} \mu-\hat{\nabla}_{\bar{\mu}} \mu\right)=\frac{1}{4} \Phi\left(\operatorname{trace}_{g_{M}} S^{\prime}\right)-\frac{n i}{2} \cos \theta H$. Therefore,

$$
\Phi\left(\frac{(1-n)}{4} \nabla \log \sin ^{2} \theta\right)=\frac{1}{4}\left(2 n(J H)^{\perp}-\Phi\left(\text { Trace }_{g_{M}} S^{\prime}\right)\right) .
$$


Using Lemma 3.1, (3.2), and $\Phi(\mu)=J d F(\mu)-i \cos \theta d F(\mu)$, we have $\Phi\left(\operatorname{Trace}_{g_{M}} S^{\prime}\right)$

$$
\begin{aligned}
= & \sum_{j, k} \hat{g}\left(S^{\prime}\left(e_{j}, e_{j}\right), \frac{e_{k}}{\sin \theta}\right) \Phi\left(\frac{e_{k}}{\sin \theta}\right) \\
= & \sum_{j, k}-\hat{g}\left(T^{\prime}\left(e_{j}, \frac{e_{k}}{\sin \theta}\right), e_{j}\right) \Phi\left(\frac{e_{k}}{\sin \theta}\right) \\
= & \frac{-4}{\sin ^{2} \theta} \sum_{\mu, \beta}\left(\left(\hat{g}\left(T^{\prime}(\mu, \beta), \bar{\mu}\right)+\hat{g}\left(T^{\prime}(\bar{\mu}, \beta), \mu\right)\right) \Phi(\bar{\beta})\right. \\
& \left.+\left(\hat{g}\left(T^{\prime}(\mu, \bar{\beta}), \bar{\mu}\right)+\hat{g}\left(T^{\prime}(\bar{\mu}, \bar{\beta}), \mu\right)\right) \Phi(\beta)\right) \\
= & -\frac{4}{\sin ^{2} \theta} \sum_{\mu, \beta}\left(g\left(\Phi\left(T^{\prime}(\bar{\mu}, \beta)\right), \Phi(\mu)\right) \Phi(\bar{\beta})+g\left(\Phi\left(T^{\prime}(\mu, \bar{\beta})\right), \Phi(\bar{\mu})\right) \Phi(\beta)\right) \\
= & \frac{8 i \cos \theta}{\sin ^{2} \theta} \sum_{\mu, \beta}\left(g\left(\nabla_{\bar{\mu}} d F(\beta), J d F(\mu)\right) \Phi(\bar{\beta})-g\left(\nabla_{\mu} d F(\bar{\beta}), J d F(\bar{\mu})\right) \Phi(\beta)\right) .
\end{aligned}
$$

Writing $(J H)^{\perp}$ in terms of $\Phi(\beta)$ and $\Phi(\bar{\beta})$,

$$
\begin{aligned}
2 n(J H)^{\perp}= & \sum_{\beta} \frac{4 n}{\sin ^{2} \theta}(g(J H, \Phi(\beta)) \Phi(\bar{\beta})+g(J H, \Phi(\bar{\beta})) \Phi(\beta)) \\
= & \sum_{\beta, \mu} \frac{8 i \cos \theta}{\sin ^{2} \theta}\left(g\left(\nabla_{\bar{\mu}} d F(\mu), J d F(\beta)\right) \Phi(\bar{\beta})\right. \\
& \left.-g\left(\nabla_{\bar{\mu}} d F(\mu), J d F(\bar{\beta})\right) \Phi(\beta)\right),
\end{aligned}
$$

and substituing these equations into (3.6), we prove Proposition 3.2.

3.1. The Weitzenböck formula for $F^{*} \omega$. For simplicity let us use the notation

$$
g_{X} Y Z=g\left(\nabla_{X} d F(Y), J d F(Z)\right) .
$$

We also observe that, from

$$
\forall \mu \quad \frac{i}{2} \cos \theta=F^{*} \omega(\mu, \bar{\mu}),
$$

valid on an open set, and from (2.4), we obtain $\forall \mu$

$$
\begin{aligned}
\frac{i}{2} d \cos \theta(X) & =d\left(F^{*} \omega(\mu, \bar{\mu})\right)(X) \\
& =\nabla_{X} F^{*} \omega(\mu, \bar{\mu})+F^{*} \omega\left(\nabla_{X} \mu, \bar{\mu}\right)+F^{*} \omega\left(\mu, \nabla_{X} \bar{\mu}\right) \\
& =-g_{X} \mu \bar{\mu}+g_{X} \bar{\mu} \mu+2\left(\left\langle\nabla_{X} \mu, \bar{\mu}\right\rangle+\left\langle\nabla_{X} \bar{\mu}, \mu\right\rangle\right) F^{*} \omega(\mu, \bar{\mu}) \\
& \left.=-g_{X} \mu \bar{\mu}+g_{X} \bar{\mu} \mu \quad \text { (no sumation over } \mu\right) .
\end{aligned}
$$


Then (3.3) is equivalent to $g\left(\nabla_{X} d F(\mu), J d F(\bar{\mu})\right)=g\left(\nabla_{X} d F(\bar{\mu}), J d F(\mu)\right), \forall \mu$ (or some $\mu$ ). From $J_{\omega} Z_{\alpha}=i Z_{\alpha}, J_{\omega} Z_{\bar{\alpha}}=-i Z_{\bar{\alpha}}$ and the fact that $J_{\omega}$ is $g_{M^{-}}$ orthogonal, we get, on $\Omega_{2 n}^{0}, \forall \alpha, \beta$, and $\forall v \in T M$

$$
\left\langle\nabla_{v} J_{\omega}(\alpha), \beta\right\rangle=2 i\left\langle\nabla_{v} \alpha, \beta\right\rangle, \quad\left\langle\nabla_{v} J_{\omega}(\alpha), \bar{\beta}\right\rangle=0 .
$$

Recall that, if $\xi$ is a $r+1$-form on $M, r \geq 0$, with values on a vector bundle $E$ over $M$ with a connection $\nabla^{E}$, then $\delta \xi$, the divergence of $\xi$, is the $r$-form on $M$ with values on $E$ given by

$$
\delta \xi\left(u_{1}, \ldots, u_{r}\right)=-\sum_{s} \nabla_{e_{s}}^{E} \xi\left(e_{s}, u_{1}, \ldots, u_{r}\right),
$$

where $e_{1}, \ldots, e_{m}$ is an orthonormal basis of $T_{p} M, u_{i} \in T_{p} M$, and $\nabla^{E} \xi$ is the covariant derivative of $\xi$ on $\bigwedge^{r+1} T^{*} M \otimes E$. Thus, $\delta$ is the formal adjoint of $d$ on forms (cf. [E-L]). Note that $\delta F^{*} \omega(X)=\left\langle\delta F^{*} \omega, X\right\rangle, \forall X \in T_{p} M$, considering on the left-hand side $F^{*} \omega$ a (closed) 2-form on $M$ and on the right-hand side an endomorphism of $T M$.

Proposition 3.3. Let $F$ be an immersion with equal Kähler angles and $\nabla \cos \theta$ denote the gradient with respect to $g_{M}$. On $\Omega_{2 n}^{0}$, and considering $F^{*} \omega$ an endomorphism of $T M$.

$$
\delta F^{*} \omega=(n-2) J_{\omega}(\nabla \cos \theta), \quad \cos \theta\left(\delta J_{\omega}\right)=(n-1) J_{\omega}(\nabla \cos \theta) .
$$

Thus,

(i) For $n=1, \delta J_{\omega}=0$ (obviously!), and $\delta F^{*} \omega=0$ iff $\theta$ is constant.

(ii) For $n=2, \delta F^{*} \omega=0$. Moreover, $\delta J_{\omega}=0$ iff $\theta$ is constant.

(iii) For $n \neq 1,2, \delta F^{*} \omega=0$ iff $\delta J_{\omega}=0$ iff $\theta$ is constant.

In particular, if $n \geq 2$ and $\left(\Omega_{2 n}^{0}, J_{\omega}, g\right)$ is Kähler, then $\theta$ is constant.

Proof. Considering $F^{*} \omega$ a 2 -form on $M$, using the symmetry of $\nabla d F$ and (2.4), if $X \in T_{p} M$,

$$
\begin{aligned}
\delta\left(F^{*} \omega\right)(X) & =\sum_{\mu}-2 \nabla_{\mu} F^{*} \omega(\bar{\mu}, X)-2 \nabla_{\bar{\mu}} F^{*} \omega(\mu, X) \\
& =\sum_{\mu} 2 g_{\mu} \bar{\mu} X-2 g_{\mu} X \bar{\mu}+2 g_{\bar{\mu}} \mu X-2 g_{\bar{\mu}} X \mu \\
& =2 \sum_{\mu}\left(-g_{X} \mu \bar{\mu}+g_{X} \bar{\mu} \mu\right)-4 \sum_{\mu}\left(g_{\bar{\mu}} X \mu-g_{\bar{\mu}} \mu X\right) .
\end{aligned}
$$

From (3.8), $\frac{n i}{2} d \cos \theta(X)=\sum_{\mu}-g_{X} \mu \bar{\mu}+g_{X} \bar{\mu} \mu$. Therefore,

$$
\delta\left(F^{*} \omega\right)(X)=n i d \cos \theta(X)-4 \sum_{\mu} \nabla_{\bar{\mu}} F^{*} \omega(\mu, X) .
$$


Since $F^{*} \omega$ is of type $(1,1)$ with respect to $J_{\omega}$, and $\forall Z \in T_{p}^{c} M, \forall \mu, \beta$, $\left\langle\nabla_{Z} \beta, \mu\right\rangle=-\left\langle\beta, \nabla_{Z} \mu\right\rangle$, we get using $(3.9)$

$$
\begin{aligned}
\nabla_{Z} F^{*} \omega(\mu, \beta) & =d\left(F^{*} \omega(\mu, \beta)\right)(Z)-F^{*} \omega\left(\nabla_{Z} \mu, \beta\right)-F^{*} \omega\left(\mu, \nabla_{Z} \beta\right) \\
& =2 i \cos \theta\left\langle\nabla_{Z} \mu, \beta\right\rangle=\cos \theta\left\langle\nabla_{Z} J_{\omega}(\mu), \beta\right\rangle .
\end{aligned}
$$

Note that, since $J_{\omega}^{2}=-\mathrm{Id}, \nabla_{X} J_{\omega}\left(J_{\omega} Y\right)=-J_{\omega}\left(\nabla_{X} J_{\omega}(Y)\right), \forall X, Y \in T_{p} M$. So

$$
\begin{aligned}
4 \sum_{\mu} \nabla_{\bar{\mu}} J_{\omega}(\mu) & =\sum_{\mu} \nabla_{X_{\mu}} J_{\omega}\left(X_{\mu}\right)+\nabla_{Y_{\mu}} J_{\omega}\left(Y_{\mu}\right)+i \nabla_{Y_{\mu}} J_{\omega}\left(X_{\mu}\right)-i \nabla_{X_{\mu}} J_{\omega}\left(Y_{\mu}\right) \\
& =-\delta J_{\omega}+i \sum_{\mu}\left(-\nabla_{X_{\mu}} J_{\omega}\left(J_{\omega} X_{\mu}\right)-\nabla_{Y_{\mu}} J_{\omega}\left(J_{\omega} Y_{\mu}\right)\right) \\
& =-\left(\delta J_{\omega}+i J_{\omega}\left(\delta J_{\omega}\right)\right) .
\end{aligned}
$$

Hence, from (3.11), and since $J_{\omega}$ is $g_{M}$-orthogonal, $\forall \beta$

$$
\sum_{\mu} \nabla_{\bar{\mu}} F^{*} \omega(\mu, \beta)=-\frac{\cos \theta}{4}\left\langle\delta J_{\omega}+i J_{\omega}\left(\delta J_{\omega}\right), \beta\right\rangle=-\frac{\cos \theta}{2}\left\langle\delta J_{\omega}, \beta\right\rangle .
$$

Moreover, $i d \cos \theta(\beta)=d \cos \theta\left(J_{\omega} \beta\right)=\left\langle\nabla \cos \theta, J_{\omega} \beta\right\rangle=-\left\langle J_{\omega}(\nabla \cos \theta), \beta\right\rangle$. From (3.10), $\delta F^{*} \omega(\beta)=\left\langle-n J_{\omega}(\nabla \cos \theta)+2 \cos \theta \delta J_{\omega}, \beta\right\rangle$. Thus, if we consider $F^{*} \omega$ an endomorphism of $T M$, and since $\langle\rangle,, J_{\omega}$, and $F^{*} \omega$ are real operators,

$$
\delta F^{*} \omega=-n J_{\omega}(\nabla \cos \theta)+2 \cos \theta \delta J_{\omega} .
$$

On the other hand, $F^{*} \omega=\cos \theta J_{\omega}$. Then, an elementary computation gives

$$
\delta F^{*} \omega=-J_{\omega}(\nabla \cos \theta)+\cos \theta \delta J_{\omega} .
$$

Comparing (3.12) with (3.13) we get the Proposition.

If we apply the Weitzenböck formula to the 2-form $F^{*} \omega$, for an immersion $F: M \rightarrow N$ we get (see e.g., [E-L] (1.32))

$$
\frac{1}{2} \triangle\left\|F^{*} \omega\right\|^{2}=-\left\langle\triangle F^{*} \omega, F^{*} \omega\right\rangle+\left\|\nabla F^{*} \omega\right\|^{2}+\left\langle S F^{*} \omega, F^{*} \omega\right\rangle,
$$

where $\langle$,$\rangle denotes the Hilbert-Schmidt inner product for 2$-forms, and $S$ is the Ricci operator of $\bigwedge^{2} T^{*} M$. We note that we use the the sign convention $\triangle \phi=+\operatorname{Trace}_{g_{M}}$ Hess $\phi$, for $\phi$ a smooth real map on $M$. This sign is opposite to the one of $[\mathbf{E}-\mathbf{L}]$, but here we use the same sign as in $[\mathbf{E}-\mathbf{L}]$ for the Laplacian of forms $\triangle=d \delta+\delta d$. If $\bar{R}$ denotes the curvature tensor of $\bigwedge^{2} T^{*} M$, and $X, Y, u, v \in T_{p} M, \xi \in \bigwedge^{2} T_{p}^{*} M$, then

$$
\begin{aligned}
& \bar{R}(X, Y) \xi(u, v)=-\xi\left(R^{M}(X, Y) u, v\right)-\xi\left(u, R^{M}(X, Y) v\right), \\
& S F^{*} \omega(X, Y)=\sum_{1 \leq i \leq 2 n}-\bar{R}\left(e_{i}, X\right) F^{*} \omega\left(e_{i}, Y\right)+\bar{R}\left(e_{i}, Y\right) F^{*} \omega\left(e_{i}, X\right),
\end{aligned}
$$


where $R^{M}$ denotes the curvature tensor of $M$. In general, we use the following sign convention for curvature tensors: $R^{M}(X, Y) Z=-\nabla_{X} \nabla_{Y} Z+$ $\nabla_{Y} \nabla_{X} Z+\nabla_{[X, Y]} Z$. Then, $R^{M}(X, Y, Z, W)=g_{M}\left(R^{M}(X, Y) Z, W\right)$. It is straightforward to prove:

Lemma 3.2. If $\left\{X_{\alpha}, Y_{\alpha}\right\}$ is a diagonalizing orthonormal basis of $F^{*} \omega$ at $p$,

$$
\begin{aligned}
\left\langle S F^{*} \omega, F^{*} \omega\right\rangle= & \sum_{\mu} 4 \cos ^{2} \theta_{\mu} \operatorname{Ricci}^{M}(\mu, \bar{\mu})+\sum_{\mu, \rho} 8 \cos \theta_{\mu} \cos \theta_{\rho} R^{M}(\rho, \bar{\rho}, \mu, \bar{\mu}) \\
= & \sum_{\mu, \rho} 4\left(\cos \theta_{\mu}+\cos \theta_{\rho}\right)^{2} R^{M}(\rho, \mu, \bar{\rho}, \bar{\mu}) \\
& +4\left(\cos \theta_{\mu}-\cos \theta_{\rho}\right)^{2} R^{M}(\bar{\rho}, \mu, \rho, \bar{\mu}) .
\end{aligned}
$$

In particular, if $F$ has equal Kähler angles at $p$, then, at $p$,

$$
\left\langle S F^{*} \omega, F^{*} \omega\right\rangle=16 \cos ^{2} \theta \sum_{\rho, \mu} R^{M}(\rho, \mu, \bar{\rho}, \bar{\mu}) .
$$

Moreover, if $\left(M, J_{\omega}, g_{M}\right)$ is Kähler in a neighbourhood of $p$, then $\left\langle S F^{*} \omega, F^{*} \omega\right\rangle$ $=0$.

We recall the concept of nonnegative isotropic sectional curvature, for $M$ with dimension $\geq 4$, defined by Micallef and Moore in [Mi-Mo]. Let

$$
K_{\text {isot }}(\sigma)=\frac{R^{M}(z, w, \bar{z}, \bar{w})}{\|z \wedge w\|^{2}}
$$

where $\sigma=\operatorname{span}_{\mathbb{C}}\{z, w\}$ is a totally isotropic complex two-plane in $T^{c} M$, that is, $u \in \sigma \Rightarrow g_{M}(u, u)=0$, and where $R^{M}(x, y, u, v)$ is extend to the complexified tangent space by $\mathbb{C}$-multilinearity. The curvature of $M$ is said to be nonnegative (resp. positive) on totally isotropic two-planes at $p$, if $K_{\text {isot }}(\sigma) \geq 0$ (resp. $>0$ ) whenever $\sigma \subset T_{p}^{c} M$ is a totally isotropic two-plane over $p$. If $M$ is compact, simply connected and has positive isotropic sectional curvature everywhere, then $M$ is homeomorphic to a sphere ([Mi-Mo]). If $n \geq 1$, $T^{2 n}$ is the flat torus, and $S^{2}$ is the euclidean sphere of $\mathbb{R}^{3}$, then $S^{2} \times T^{2 n}$, $S^{2} \times S^{2}, S^{2} \times S^{2} \times T^{2 n}$ and the complex projective space $\mathbb{C P}^{n}$ have isotropic sectional curvature $\geq 0$ but not $>0$. If $\left\{X_{\alpha}, Y_{\alpha}\right\}$ is any orthonormal basis of $T_{p} M$, and " $\mu$ " denotes $Z_{\mu}$ as in (1.3), the expression

$$
S_{\text {isot }}\left(\left\{Z_{\alpha}\right\}_{1 \leq \alpha \leq n}\right)=\sum_{\rho \neq \mu} K_{\text {isot }}\left(\operatorname{span}_{\mathbb{C}}\{\rho, \mu\}\right)=4 \sum_{\rho, \mu} R^{M}(\rho, \mu, \bar{\rho}, \bar{\mu})
$$

is a hermitian trace of the curvature of $M$ restricted to the maximal totally isotropic subspace $\operatorname{span}_{\mathbb{C}}\left\{Z_{1}, \ldots, Z_{n}\right\}$ of $T^{c} M$. To require it to be $\geq 0$, for all maximal totally isotropic subspaces - and we will say that $M$ has nonnegative isotropic scalar curvature - seems, for $n \geq 2$, to be strictly weaker than to have nonnegative isotropic sectional curvature. We also note that, any other metric conformaly equivalent to the flat metric $g_{0}$ on 
the 2n-torus with nonnegative isotropic scalar curvature is homothetically equivalent to $g_{0}$, hence flat. In fact, in general, if $\hat{g}=e^{\phi} g_{M}$ is a conformaly equivalent metric on $M$, then, for each $g_{M}$-orthonormal basis $\left\{X_{\alpha}, Y_{\alpha}\right\}$, $\hat{S}_{\text {isot }}\left(\left\{\hat{Z}_{\alpha}\right\}\right)=e^{-\phi} S_{\text {isot }}\left(\left\{Z_{\alpha}\right\}\right)-(n-1) e^{-2 \phi}\left(2 \triangle \phi+(n-1)\|\nabla \phi\|^{2}\right)$, where $\hat{Z}_{\alpha}$ are defined by the $\hat{g}$-orthonormal basis $\left\{e^{-\frac{\phi}{2}} X_{\alpha}, e^{-\frac{\phi}{2}} Y_{\alpha}\right\}$. To require $2 \triangle \phi+(n-1)\|\nabla \phi\|^{2} \leq 0$, implies, in case of $M$ compact, $\phi$ constant. We observe that, if $\operatorname{dim}_{\mathbb{R}} M \geq 6$, then $S_{\text {isot }} \equiv 0$ does not imply $M$ to be flat, but $K_{\text {isot }} \equiv 0$ implies so. We also note that, if $\operatorname{dim}_{\mathbb{R}}\left(T_{p} M\right)=4$, the set of curvature tensors at $p$, with zero isotropic sectional curvature is a vector space of dimension 9 .

Recall that, for an immersion with equal Kähler angles, $F^{*} \omega$ is parallel iff $\theta$ is constant and if $\cos \theta \neq 0,\left(M, J_{\omega}, g_{M}\right)$ is a Kähler manifold. We are going to see that an extra condition on the scalar isotropic curvature of $M$ may imply itself that the Kähler angle is constant and/or $\nabla J_{\omega}=0$. From Proposition 3.3, for any $n \geq 1$, on $\Omega_{2 n}^{0} \cup \Omega_{0}^{0}$

$$
\left\|\delta F^{*} \omega\right\|^{2}=(n-2)^{2}\|\nabla \cos \theta\|^{2} .
$$

In particular, if $n \neq 2,\|\nabla \cos \theta\|^{2}$ is smoothly extended to all $M$, and from (3.1) we get that $\cos ^{2} \theta\left\|\nabla J_{\omega}\right\|^{2}$ is also smooth. Observe that $\left\|\delta F^{*} \omega\right\|^{2}$ has the same value considering $\delta F^{*} \omega$ a vector or a 1 -form, but considering $F^{*} \omega$ a 2 -form (as in (3.14)) $\left\|\nabla F^{*} \omega\right\|^{2}$ is half of the square norm when considering $F^{*} \omega$ an operator of $T M$ (as in (3.1)). For $n=2, F^{*} \omega$ is co-closed, and so it is a harmonic 2-form. In fact, since $F$ has equal Kähler angles, $F^{*} \omega=$ $\cos \theta\left(X_{*}^{1} \wedge Y_{*}^{1}+X_{*}^{2} \wedge Y_{*}^{2}\right)$, and so $* F^{*} \omega= \pm F^{*} \omega$, where $*$ is the Hodge staroperator of $(M, g)$, for a fixed local orientation of $M$, and the \pm sign depends on the orientation of the diagonalizing basis. In particular, $F^{*} \omega$ is co-closed (on $\Omega_{0}^{0} \cup \Omega_{n}^{0}$ and so on all $M$ ). From harmonicity of $F^{*} \omega$ we may conclude that if the set of Lagrangian points has non-empty interior, or more generaly, if $F^{*} \omega$ as a zero of infinite order, then $F$ is Lagrangian (see e.g., [E-L] (1.27), (1.28)). For any $n \geq 2$, integrating (3.14) on $M$, using Stokes, (3.16) and (3.1), and the fact that $\int_{M}\left\langle\triangle F^{*} \omega, F^{*} \omega\right\rangle \operatorname{Vol}_{M}=\int_{M}\left\|\delta F^{*} \omega\right\|^{2} \mathrm{Vol}_{M}$, we have

$$
\begin{aligned}
0= & \int_{M}\left(\left(n-(n-2)^{2}\right)\|\nabla \cos \theta\|^{2}+\frac{1}{2} \cos ^{2} \theta\left\|\nabla J_{\omega}\right\|^{2}\right) \operatorname{Vol}_{M} \\
& +\int_{M}\left\langle S F^{*} \omega, F^{*} \omega\right\rangle \operatorname{Vol}_{M} .
\end{aligned}
$$

The first integrand is smooth on $M$, for all $n$ (for $n=2$ it gives half of (3.1)). The factor $n-(n-2)^{2}$ is respectively, $>0,=0,<0$, according $n=2$ or $3, n=4$, and $n \geq 5$. If $M$ has nonnegative isotropic scalar curvature, $\left\langle S F^{*} \omega, F^{*} \omega\right\rangle \geq 0$, by Lemma 3.2. Recall from Proposition 3.3 that $\left(\Omega_{2 n}^{0}, J_{\omega}, g\right)$ to be Kähler is a sufficient condition to conclude $\theta$ is constant. Then we conclude: 
Proposition 3.4. Let $F$ be a non-Lagrangian immersion with equal Kähler angles of a compact orientable $M$ with nonnegative isotropic scalar curvature into a Kähler manifold $N$. If $n=2,3$ or 4 , then $\theta$ is constant and $\left(M, J_{\omega}, g_{M}\right)$ is a Kähler manifold. For any $n \geq 1$ and $\theta$ constant, $F^{*} \omega$ is parallel, i.e., $\left(M, J_{\omega}, g_{M}\right)$ is a Kähler manifold.

\section{Minimal immersions with equal Kähler angles.}

Let us assume that $F: M \rightarrow N$ is minimal with equal Kähler angles. On a open set of $M \sim \mathcal{L}$ where a orthonormal frame $\left\{X_{\alpha}, Y_{\alpha}=J_{\omega}\left(X_{\alpha}\right)\right\}$ is defined, we have from (3.11) and (2.4), for any $p, Z \in T_{p} M$ and $\mu, \gamma$,

$$
2 \cos \theta\left\langle\nabla_{Z} \mu, \gamma\right\rangle=-i \nabla_{Z} F^{*} \omega(\mu, \gamma)=i g_{Z} \mu \gamma-i g_{Z} \gamma \mu .
$$

Note that $F^{*} \omega\left(\nabla_{Z} \mu, \bar{\gamma}\right)=i \cos \theta\left\langle\nabla_{Z} \mu, \bar{\gamma}\right\rangle=-i \cos \theta\left\langle\mu, \nabla_{Z} \bar{\gamma}\right\rangle=-F^{*} \omega(\mu$, $\left.\nabla_{Z} \bar{\gamma}\right)$. Hence, if $\mu \neq \gamma, \nabla_{Z} F^{*} \omega(\mu, \bar{\gamma})=d\left(F^{*} \omega(\mu, \bar{\gamma})\right)(Z)=0$. Thus

$$
g_{Z} \mu \bar{\gamma}=g_{Z} \bar{\gamma} \mu, \quad \forall \mu \neq \gamma .
$$

From (3.8), for each $\mu$,

$$
-\frac{i}{2} d \cos \theta(Z)=-\nabla_{Z} F^{*} \omega(\mu, \bar{\mu})=g_{Z} \mu \bar{\mu}-g_{Z} \bar{\mu} \mu \text { (no sumation over } \mu \text { ). }
$$

From (1.6), on $M \sim(\mathcal{L} \cup \mathcal{C})$

$$
\triangle \kappa=4 i \sum_{\beta} \operatorname{Ricci}^{N}(J d F(\beta), d F(\bar{\beta}))
$$

$$
\begin{aligned}
& +\frac{32}{\sin ^{2} \theta} \sum_{\beta, \mu} \operatorname{Im}\left(R^{N}(d F(\beta), d F(\mu), d F(\bar{\beta}), J d F(\bar{\mu})+i \cos \theta d F(\bar{\mu}))\right) \\
& -\frac{128 \cos \theta}{\sin ^{4} \theta} \sum_{\beta, \mu, \rho} \operatorname{Re}\left(g_{\beta} \mu \bar{\rho} g_{\bar{\beta}} \rho \bar{\mu}\right) \\
& +\frac{64 \cos \theta}{\sin ^{2} \theta} \sum_{\beta, \mu, \rho}\left(\left|\left\langle\nabla_{\beta} \mu, \rho\right\rangle\right|^{2}+\left|\left\langle\nabla_{\bar{\beta}} \mu, \rho\right\rangle\right|^{2}\right)
\end{aligned}
$$

where now $\kappa=n \log \left(\frac{1+\cos \theta}{1-\cos \theta}\right)$. Since $R(X, Y, Z, J W)$ is skew-symmetric on $(X, Y)$ and symmetric on $(Z, W), \sum_{\mu, \beta} R^{N}(d F(\beta), d F(\mu), d F(\bar{\beta}), J d F(\bar{\mu}))=$ 0 . Then, from the Gauss equation and minimality of $F$,

$$
\begin{aligned}
(4.4) & =\sum_{\beta, \mu} \frac{32}{\sin ^{2} \theta} \operatorname{Im}\left(i \cos \theta R^{N}(d F(\beta), d F(\mu), d F(\bar{\beta}), d F(\bar{\mu}))\right) \\
& =\frac{32 \cos \theta}{\sin ^{2} \theta} \sum_{\beta, \mu} R^{M}(\beta, \mu, \bar{\beta}, \bar{\mu})+g(\nabla d F(\beta, \bar{\mu}), \nabla d F(\mu, \bar{\beta})) .
\end{aligned}
$$


Using the unitary basis $\left\{\frac{\sqrt{2}}{\sin \theta} \Phi(\rho), \frac{\sqrt{2}}{\sin \theta} \Phi(\bar{\rho})\right\}$ of the normal bundle,

$$
\begin{aligned}
& \frac{32 \cos \theta}{\sin ^{2} \theta} \sum_{\beta, \mu} g(\nabla d F(\beta, \bar{\mu}), \nabla d F(\mu, \bar{\beta})) \\
& =\frac{64 \cos \theta}{\sin ^{4} \theta} \sum_{\beta, \mu, \rho}\left(\left|g_{\beta} \bar{\mu} \rho\right|^{2}+\left|g_{\beta} \overline{\mu \rho}\right|^{2}\right) \\
& =\frac{64 \cos \theta}{\sin ^{4} \theta} \sum_{\beta, \mu, \rho}\left(\left|g_{\beta} \bar{\rho} \mu\right|^{2}+\left|g_{\bar{\mu}} \beta \bar{\rho}\right|^{2}\right)=\frac{128 \cos \theta}{\sin ^{4} \theta} \sum_{\beta, \mu, \rho}\left|g_{\beta} \bar{\rho} \mu\right|^{2} .
\end{aligned}
$$

From (4.2) and (4.3),

$$
\begin{aligned}
& \sum_{\beta, \mu, \rho} \operatorname{Re}\left(g_{\beta} \mu \bar{\rho} g_{\bar{\beta}} \rho \bar{\mu}\right)=\sum_{\beta, \mu} \sum_{\rho \neq \mu}\left|g_{\beta} \bar{\rho} \mu\right|^{2}+\sum_{\beta, \mu} \operatorname{Re}\left(g_{\beta} \mu \bar{\mu} g_{\bar{\beta}} \mu \bar{\mu}\right) \\
& =\sum_{\beta, \mu, \rho}\left|g_{\beta} \bar{\rho} \mu\right|^{2}-\sum_{\beta, \mu}\left|g_{\beta} \bar{\mu} \mu\right|^{2}+\sum_{\beta, \mu} \operatorname{Re}\left(g_{\beta} \mu \bar{\mu} g_{\bar{\beta}} \mu \bar{\mu}\right) \\
& =\sum_{\beta, \mu, \rho}\left|g_{\beta} \bar{\rho} \mu\right|^{2}-\sum_{\beta, \mu} \operatorname{Re}\left(\frac{i}{2} d \cos \theta(\beta) g_{\bar{\beta}} \mu \bar{\mu}\right),
\end{aligned}
$$

so

$$
(4.7)+(4.5)=\frac{128 \cos \theta}{\sin ^{4} \theta} \sum_{\beta, \mu} \operatorname{Re}\left(\frac{i}{2} d \cos \theta(\beta) g_{\bar{\beta}} \mu \bar{\mu}\right) .
$$

On the other hand, Proposition 3.2 and minimality of $F$ gives,

$$
-\sum_{\beta, \mu} \frac{4 \cos \theta}{\sin ^{2} \theta} \operatorname{Re}\left(i g_{\beta} \bar{\mu} \mu \cdot \bar{\beta}\right)=\frac{1-n}{4} \nabla \log \sin ^{2} \theta=\frac{(n-1) \cos \theta}{2 \sin ^{2} \theta} \nabla \cos \theta .
$$

Consequentely,

$$
\begin{aligned}
& \frac{128 \cos \theta}{\sin ^{4} \theta} \sum_{\beta, \mu} \operatorname{Re}\left(\frac{i}{2} d \cos \theta(\beta) g_{\bar{\beta}} \mu \bar{\mu}\right) \\
& =\frac{128 \cos \theta}{\sin ^{4} \theta} \sum_{\beta, \mu} \operatorname{Re}\left(-\frac{i}{2} d \cos \theta(\bar{\beta}) g_{\beta} \bar{\mu} \mu\right) \\
& =-\frac{64 \cos \theta}{\sin ^{4} \theta} d \cos \theta\left(\operatorname{Re}\left(\sum_{\beta, \mu} i g_{\beta} \bar{\mu} \mu \cdot \bar{\beta}\right)\right)=\frac{8(n-1) \cos \theta}{\sin ^{4} \theta}\|\nabla \cos \theta\|^{2} .
\end{aligned}
$$

That is,

$$
(4.7)+(4.5)=\frac{8(n-1) \cos \theta}{\sin ^{4} \theta}\|\nabla \cos \theta\|^{2} .
$$


Using (3.9),

$$
\begin{aligned}
\left\|\nabla J_{\omega}\right\|^{2} & =\sum_{\beta} 4\left\langle\nabla_{\beta} J_{\omega}, \nabla_{\bar{\beta}} J_{\omega}\right\rangle \\
& =\sum_{\beta} \sum_{\mu, \rho} 16\left(\left|\left\langle\nabla_{\beta} J_{\omega}(\mu), \rho\right\rangle\right|^{2}+\left|\left\langle\nabla_{\beta} J_{\omega}(\bar{\mu}), \bar{\rho}\right\rangle\right|^{2}\right) \\
& =64 \sum_{\beta, \mu, \rho}\left(\left|\left\langle\nabla_{\beta} \mu, \rho\right\rangle\right|^{2}+\left|\left\langle\nabla_{\bar{\beta}} \mu, \rho\right\rangle\right|^{2}\right) .
\end{aligned}
$$

Thus we see that $(4.6)=\frac{\cos \theta}{\sin ^{2} \theta}\left\|\nabla J_{\omega}\right\|^{2}$. So we have obtained the following formula:

Proposition 4.1. If $N$ is Kähler-Einstein with Ricci tensor $\operatorname{Ricci}^{N}=R g$, and $F$ is a minimal immersion with equal Kähler angles, on an open set without complex and Lagrangian points,

$$
\begin{aligned}
\triangle \kappa= & \cos \theta\left(-2 n R+\frac{32}{\sin ^{2} \theta} \sum_{\beta, \mu} R^{M}(\beta, \mu, \bar{\beta}, \bar{\mu})\right. \\
& \left.+\frac{1}{\sin ^{2} \theta}\left\|\nabla J_{\omega}\right\|^{2}+\frac{8(n-1)}{\sin ^{4} \theta}\|\nabla \cos \theta\|^{2}\right) .
\end{aligned}
$$

Note that if $n=1$ we get the expression of Wolfson [W], $\triangle \kappa=-2 R \cos \theta$. Proposition 4.2. If $N$ is Kähler-Einstein with Ricci tensor $\operatorname{Ricci}^{N}=R g$, and $F$ is a minimal immersion with equal Kähler angles, then:

(i) If $n=2$,

$$
R \sin ^{2} \theta \cos ^{2} \theta=0 .
$$

(ii) If $n \geq 3$, then $\|\nabla|\sin \theta|\|^{2}$ can be smoothly extended to all $M$. Moreover, if $M$ is compact and orientable,

$$
\begin{aligned}
& \int_{M} n R \sin ^{2} \theta \cos ^{2} \theta \mathrm{Vol}_{M} \\
& =\int_{M}\left((n-2)^{2}\|\nabla \cos \theta\|^{2}+2(n-2)\|\nabla|\sin \theta|\|^{2}\right) \operatorname{Vol}_{M} .
\end{aligned}
$$

Proof. Multiplying (4.10) by $\sin ^{2} \theta \cos \theta$, we get, on $M \sim \mathcal{C} \cup \mathcal{L}$, and using Lemma 3.2,

$$
\begin{aligned}
\sin ^{2} \theta \cos \theta \triangle \kappa= & -2 n \sin ^{2} \theta \cos ^{2} \theta R+2\left\langle S F^{*} \omega, F^{*} \omega\right\rangle \\
& +\cos ^{2} \theta\left\|\nabla J_{\omega}\right\|^{2}+\frac{8(n-1) \cos ^{2} \theta}{\sin ^{2} \theta}\|\nabla \cos \theta\|^{2} .
\end{aligned}
$$


On the other hand, $\kappa=n \log \left(\frac{1+\cos \theta}{1-\cos \theta}\right)$, and so, $\triangle \kappa=\frac{2 n}{\sin ^{2} \theta} \triangle \cos \theta+$ $\frac{4 n \cos \theta}{\sin ^{4} \theta}\|\nabla \cos \theta\|^{2}$. Hence,

$$
\begin{aligned}
& 2 n \cos \theta \triangle \cos \theta+\frac{4 n \cos ^{2} \theta}{\sin ^{2} \theta}\|\nabla \cos \theta\|^{2} \\
& =-2 n \sin ^{2} \theta \cos ^{2} \theta R+2\left\langle S F^{*} \omega, F^{*} \omega\right\rangle \\
& \quad+\cos ^{2} \theta\left\|\nabla J_{\omega}\right\|^{2}+\frac{8(n-1) \cos ^{2} \theta}{\sin ^{2} \theta}\|\nabla \cos \theta\|^{2} .
\end{aligned}
$$

Recall that, from (3.1), and considering $F^{*} \omega$ a 2 -form, $\left\|\nabla F^{*} \omega\right\|^{2}=$ $\frac{1}{2} \cos ^{2} \theta\left\|\nabla J_{\omega}\right\|^{2}+n\|\nabla \cos \theta\|^{2}$. Since $\triangle \cos ^{2} \theta=2 \cos \theta \triangle \cos \theta+2\|\nabla \cos \theta\|^{2}$, substituting this into (4.13), we have

$$
\begin{aligned}
n \triangle \cos ^{2} \theta= & -2 n \sin ^{2} \theta \cos ^{2} \theta R+2\left\langle S F^{*} \omega, F^{*} \omega\right\rangle \\
& +2\left\|\nabla F^{*} \omega\right\|^{2}+\frac{4(n-2) \cos ^{2} \theta}{\sin ^{2} \theta}\|\nabla \cos \theta\|^{2}
\end{aligned}
$$

and, for $n=2$,

$$
n \triangle \cos ^{2} \theta=-2 n \sin ^{2} \theta \cos ^{2} \theta R+2\left\langle S F^{*} \omega, F^{*} \omega\right\rangle+2\left\|\nabla F^{*} \omega\right\|^{2} .
$$

Let us now suppose that $n \geq 3$. The $\operatorname{sign}$ of $\sin \theta$ is not determined, because we have chosen the interval where $\theta$ is such that $\cos \theta \geq 0$. Nevertheless we have $|\sin \theta|=\sqrt{1-\cos \theta} \sqrt{1+\cos \theta}$. This map is continuous, smooth on $M \sim \mathcal{C} \cup \mathcal{L}$ but could be not Lipschitz near complex points. The last term of (4.14) is given by

$$
\begin{aligned}
\frac{4(n-2) \cos ^{2} \theta}{\sin ^{2} \theta}\|\nabla \cos \theta\|^{2} & =(n-2) \frac{\left\|\nabla \cos ^{2} \theta\right\|^{2}}{\sin ^{2} \theta} \\
& =(n-2) \frac{\left\|\nabla \sin ^{2} \theta\right\|^{2}}{\sin ^{2} \theta}=4(n-2)\|\nabla \mid \sin \theta\|^{2} .
\end{aligned}
$$

Then (4.14) is the equation

$$
\begin{aligned}
n \triangle \cos ^{2} \theta= & -2 n \sin ^{2} \theta \cos ^{2} \theta R+2\left\langle S F^{*} \omega, F^{*} \omega\right\rangle \\
& +2\left\|\nabla F^{*} \omega\right\|^{2}+4(n-2)\|\nabla|\sin \theta|\|^{2} .
\end{aligned}
$$

Clearly, (4.16) is valid on $\Omega_{2 n}^{0} \sim \mathcal{C}$ and also on $\Omega_{0}^{0}$ and at interior points of $\mathcal{C}$. From smoothness over all $M$ of all terms but the last, and the fact that the remaining set is a set of Lagrangian and complex points with no interior, formula (4.16) is valid on all $M$, extending smoothly and nonnegatively $\|\nabla|\sin \theta|\|^{2}$. Integrating it over $M$, and using (3.17) and (3.1), we have

$$
\begin{aligned}
\int_{M} 2 n R \sin ^{2} \theta \cos ^{2} \theta \operatorname{Vol}_{M}= & \int_{M}\left(-2\left(n-(n-2)^{2}\right)+2 n\right)\|\nabla \cos \theta\|^{2} \\
& +4(n-2)\|\nabla|\sin \theta|\|^{2} \operatorname{Vol}_{M},
\end{aligned}
$$


leading to (4.12). If $n=2$, we see that (4.15) is also valid at Lagrangian and complex points. In fact all terms of (4.15) vanish at interior points of the Lagrangian and complex sets (see Lemma 3.2 and (3.1)). Since they are smooth on all $M$, they must vanish at boundary points of $\mathcal{C}$ and of $\mathcal{L}$. Thus, the above equation is valid on all $M$, with or without complex or Lagrangian points. Now, (4.11) follows from (4.15), and use of (3.14) with $\left\|F^{*} \omega\right\|^{2}=n \cos ^{2} \theta$ and $\triangle F^{*} \omega=0$.

Proof of Theorem 1.2 and Theorem 1.3. If $n=2$ and $R \neq 0$, (4.11) implies $\sin ^{2} \theta \cos ^{2} \theta=0$. Hence $F$ is either Lagrangian or a complex submanifold. If $n \geq 3$, and $M$ is compact and oriented, the right-hand side of (4.12) is nonnegative, while the left-hand side is non-positive for $R<0$. Then, $\sin ^{2} \theta \cos ^{2} \theta=0$ must hold on all $M$, that is, $F$ is either Lagrangian or complex. If $R=0$, the right-hand side of (4.12) must vanish. Then, for $n \geq 3, \cos \theta$ must be constant, and we have proved Theorem 1.2. If $\cos \theta$ is constant, the right-hand side of (4.12) vanishes. Hence, if $R \neq 0, F$ is either complex or Lagrangian, and Theorem 1.3 is proved.

Proof of Theorem 1.4. If $M$ is not Lagrangian, under the curvature condition on $M$, by Proposition 3.4 , for $n=2,3$ or $4,\left(M, J_{\omega}, g_{M}\right)$ is a Kähler manifold and $\cos \theta$ is constant. So, if $M$ is not a complex submanifold, by (4.11), or (4.12), $R=0$. In general, if $n \geq 1$ and $\theta$ is constant, Proposition 3.4 also applies.

Under the conditions of Proposition 3.4, if $M$ is homeomorphic to a 4 or 6 dimensional sphere, immersed into a Kähler-Einstein manifold, with equal Kähler angles and with nonnegative isotropic scalar curvature, then it must be Lagrangian, for it is well-known that such manifolds cannot carry a Kähler structure. Obviously, any Riemannian manifold $M$ with strictly positive isotropic scalar curvature cannot carry any Kähler structure, and so the same conclusion must hold. No minimality is required to conclude this.

As an observation, Proposition 3.4 should be compared with the following lemma:

Lemma 4.1. Let $F$ be an immersion with equal Kähler angles, and $n \geq 2$. If $\cos \theta$ is constant then:

(i) $(A, B, C) \rightarrow g_{A} B C$ is symmetric whenever $A, B$, and $C$ are not all of the same type.

(ii) $\left\langle\nabla_{\bar{\beta}} \mu, \gamma\right\rangle=0, \forall \beta, \mu, \gamma$.

(iii) $F^{*} \omega$ is an harmonic 2 -form of constant norm.

(iv) $32 \sum_{\beta, \mu} R^{M}(\beta, \mu, \bar{\beta}, \bar{\mu})=-64 \sum_{\beta, \mu, \rho}\left|\left\langle\nabla_{\beta} \mu, \rho\right\rangle\right|^{2}=-\left\|\nabla J_{\omega}\right\|^{2} \leq 0$ (only in the case $\cos \theta \neq 0$ ). 
Proof. Since $\cos \theta$ is constant, we obtain $(4.3)=0$. This, together (4.2), and the symmetry of $\nabla d F$, proves (i). But (i) and (4.1) imply (ii). (iii) comes from (3.16) and that $\left\|F^{*} \omega\right\|^{2}=n \cos ^{2} \theta$. Now we prove (iv). Since $F^{*} \omega$ is harmonic, from Weitzenböck formula (3.14) we conclude $\left\langle S F^{*} \omega, F^{*} \omega\right\rangle=$ $-\left\|\nabla F^{*} \omega\right\|^{2}$. Lemma 3.2 and (3.1) (but considering $F^{*} \omega$ a 2-form) gives (iv).

Remark 2. If $N$ is a Kähler manifold of constant holomorphic sectional curvature equal to $K$ (and so $R=2(2 n+1) K$ ), and the isotropic scalar curvature of $M$ satisfies $S_{\text {isot }} \geq c, c$ a constant, we get by Gauss equation, that $c \leq \frac{n(n-1) K}{4}$. Thus, nonnegative isotropic scalar curvature on $M$ is a possible condition for $K \geq 0$. In the case $K=0$, that is, $N$ is the flat complex torus, for $n \geq 2, F$ must be totally geodesic, and so $M$ is flat. We also note that $S_{\text {isot }}>\frac{n}{4}$ is not a possible condition if $K>0$. Such a condition, when possible (and so $N$ cannot be of constant holomorphic sectional curvature), could lead to some conclusion by applying the maximum principle to (4.10) at a maximum point of $\kappa$.

Example. Let $(N, I, J, g)$ be an hyper-Kähler manifold of real dimension 8. Thus, $I$ and $J$ are two $g$-orthogonal complex structures on $N$, such that $I J=-J I$ and $\nabla I=\nabla J=0$, where $\nabla$ is the Levi-Civita connection relative to $g$. It is known that such manifolds are Ricci-flat $([\mathbf{B}])$. Set $K=I J$. For each $\nu, \phi$, we take " $\nu \phi "=(\cos \nu, \sin \nu \cos \phi, \sin \nu \sin \phi) \in S^{2}$, and define $J_{\nu \phi}=\cos \nu I+\sin \nu \cos \phi J+\sin \nu \sin \phi K$. These $J_{\nu \phi}$ are the complex structures on $N$ compatible with its hyper-Kähler structure, that is, they are $g$-orthogonal and $\nabla J_{\nu \phi}=0$.

Two of such complex structures, $J_{\nu \phi}$ and $J_{\mu \rho}$, anti-commute at a point $p$ iff $J_{\nu \phi}(X)$ and $J_{\mu \rho}(X)$ are orthogonal for some nonzero $X \in T_{p} N$, iff $\nu \phi$ and $\mu \rho$ are orthogonal in $\mathbb{R}^{3}$. Thus, they anti-commute at a point $p$ iff they anti-commute everywhere. If that is the case $J_{\nu \phi} \circ J_{\mu \rho}=J_{\sigma \epsilon}$, where $\{\nu \phi, \mu \rho, \sigma \epsilon\}$ is a direct orthonormal basis of $\mathbb{R}^{3}$. For each unit vector $X \in$ $T_{p} N$, set $H_{X}=\operatorname{span}\{X, I X, J X, K X\}=\operatorname{span}\left\{X, J_{\nu \phi}(X), J_{\mu \rho}(X), J_{\sigma \epsilon}(X)\right\}$, for any orthonormal basis $\{\nu \phi, \mu \rho, \sigma \epsilon\}$. If $Y \in H_{X}^{\perp}$ is another unit vector, then $H_{X} \perp H_{Y}$. Let $\omega_{\nu \phi}$ be the Kähler form of $\left(N, J_{\nu \phi}, g\right)$. Let $E$ be a 4dimensional vector subspace of $T_{p} N$. We first note that $E=H_{X}$ for some $X \in E$, iff $J_{\nu \phi}(E) \subset E$ for any $\nu, \phi$. If that is the case, then $E$ is not a Lagrangian subspace with respect to any complex structure $J_{\mu \rho}$. In general, $E$ contains a $J_{\nu \phi}$-complex line for some $\nu \phi$ iff $\operatorname{dim}\left(E \cap H_{X}\right) \geq 2$ for some $X \in$ $E$. If that is the case, and if $E$ is a Lagrangian subspace of $T_{p} N$ with respect to $J_{\mu \rho}$, then $\nu \phi \perp \mu \rho$. Furthermore, if $E$ is a $J_{\nu \phi}$-complex subspace, then $E$ is $J_{\mu \rho}$-Lagrangian iff there exist an orthonormal basis $\left\{X, J_{\nu \phi} X, Y, J_{\nu \phi} Y\right\}$ of $E$ with $H_{X} \perp H_{Y}$. To see this, let us suppose $E$ is $J_{\nu \phi^{-}}$complex subspace and $J_{\mu \rho}$-Lagrangian. We take $\left\{X, J_{\nu \phi} X, Y, J_{\nu \phi} Y\right\}$ an ortonormal basis of $E$. 
Then $Y \in \operatorname{span}\left\{X, J_{\nu \phi} X, J_{\mu \rho} X\right\}^{\perp}$. So $Y=t J_{\sigma \epsilon} X+\tilde{Y}$, for some $t \in \mathbb{R}$ and $\widetilde{Y} \in H_{X}^{\perp}$, and where $\{\nu \phi, \mu \rho, \sigma \epsilon\}$ is an ortonormal basis of $\mathbb{R}^{3}$. As $E \neq H_{X}$, $\widetilde{Y} \neq 0$. From $0=\left\langle J_{\mu \rho} Y, J_{\nu \phi} X\right\rangle$, we get $t=0$. Thus, $Y \in H_{X}^{\perp}$. We observe that, in general, $J_{\mu \rho}$-Lagrangian subspaces do not need to be $J_{\nu \phi}$-complex, as for example $E=\left\{X, J_{\nu \phi} X, Y, J_{\sigma \epsilon} Y\right\}$, with $Y \in H_{X}^{\perp}$, that contains two orthogonal complex lines for different complex strutures.

Any $J_{\nu \phi^{-}}$complex submanifold $F: M \rightarrow N$ of real dimension 4 , is for each $\mu \rho$, a minimal submanifold of $\left(N, J_{\mu \rho}, g\right)$ with equal Kähler angles. Moreover, if for each point $p \in M$, there exist an orthonormal basis $\left\{X, J_{\nu \phi} X, Y\right.$, $\left.J_{\nu \phi} Y\right\}$ of $T_{p} M$ with $H_{X} \perp H_{Y}$, the Kähler angle is constant, given by $\cos \theta=$ $|\langle\nu \phi, \mu \rho\rangle|$, where $\langle$,$\rangle is the inner product of \mathbb{R}^{3}$, and $\pm J_{\nu \phi}$ is the complex structure of $M$ which comes from polar decomposition of $\omega_{\mu \rho}$ restricted to $M$. In fact, such an orthonormal basis of $T_{p} M$ diagonalizes $\omega_{\mu \rho}$ restricted to $M$. Next proposition is an application of Proposition 3.4 or Theorem 1.4, for 4-dimensional submanifolds of $N$, where $\omega_{I}$ is the Kähler form of $(N, I, g)$ :

Proposition 4.3. Let $F: M \rightarrow N$ be a minimal immersion of a compact, oriented 4-dimensional submanifold with nonnegative isotropic scalar curvature, and such that $\forall \nu \phi \in S^{2}, F$ has equal Kähler angles with respect to $J_{\nu \phi}$. If $\exists p \in M$ and $\exists X \in T_{p} M$, unit vector, such that $\operatorname{dim}\left(T_{p} M \cap H_{X}\right) \geq 2$, then there exists $\nu \phi \in S^{2}$ such that $M$ is a $J_{\nu \phi}$-complex submanifold. Furthermore, if $J_{\nu \phi}=I$ then $F: M \rightarrow(N, I, g)$ is obviously pluriminimal. If $J_{\nu \phi} \neq I$ but $T_{p} M \cap H_{X}^{\perp} \neq\{0\}$, then $F^{*} \omega_{I}=\cos \nu J_{\nu \phi}$, and if $F$ is not I-Lagrangian, $F: M \rightarrow(N, I, g)$ is still pluriminimal.

Note that, if $T_{p} M=H_{X}$, then $J_{\nu \phi}$ can be chosen equal to $I$. The first conclusion of this result should be compared with a result of Wolfson $[\mathbf{W}]$, for $M$ a real minimal surface and $N$ a Ricci-flat K3 surface. In the latter case, there is only one Kähler angle, $\forall X \operatorname{dim}\left(T_{p} M \cap H_{X}\right)=2$ is automatically satisfied, and the isotropic scalar curvature is always zero.

Proof. From the assumption, $\operatorname{dim}\left(T_{p} M \cap H_{X}\right) \geq 2$, we may take a unit vector $Z \in T_{p} M \cap H_{X}$ such that $Z \perp X$. Then, $Z=J_{\nu \phi}(X)$ for some $\nu \phi$. Thus, $\operatorname{span}\left\{X, J_{\nu \phi}(X)\right\} \subset T_{p} M$. This implies $F^{*} \omega_{\nu \phi}\left(X, J_{\nu \phi}(X)\right)=1$. As the Kähler angles are equal, $\cos \theta_{\nu \phi}=1$ at $p$. Applying Proposition 3.4 to $F: M \rightarrow\left(N, J_{\nu \phi}, g\right), F^{*} \omega_{\nu \phi}=\cos \theta_{\nu \phi} J_{\omega_{\nu \phi}}$ with $\cos \theta_{\nu \phi}$ constant. Then $\cos \theta_{\nu \phi}=1$ everywhere. That is, $M$ is a $J_{\nu \phi}$-complex submanifold. Moreover, from the second assumption, $T_{p} M \cap H_{X}^{\perp} \neq\{0\}$, we may take a unit vector $Y \in T_{p} M \cap H_{X}^{\perp}$. Then $\left\{X, J_{\nu \phi} X, Y, J_{\nu \phi} Y\right\}$ constitutes an orthonormal basis of $T_{p} M$, that diagonalizes $F^{*} \omega_{I}$, and $F^{*} \omega_{I}=\cos \nu J_{\nu \phi}$. This means that $\nu$ or $\nu+\pi$ is the constant Kähler angle of $F: M \rightarrow(N, I, g)$, and, since $M$ is a $J_{\nu \phi^{-}}$-complex submanifold, it is pluriharmonic with respect to $\pm J_{\nu \phi}$, and so, if $\cos \nu \neq 0$, it is pluriminimal as an immersion into $(N, I, g)$. 


\section{Appendix: The computation of $\triangle \kappa$.}

We prove (1.6) for $F$ minimal and away from complex directions. First, we compute some derivative formulas of a determinant, which we will need.

Lemma 5.1. Let $A: M \rightarrow \mathcal{M}_{m \times m}(\mathbb{C})$ be a smooth map of matrices $p \rightarrow$ $A(p)=\left[A_{1}, \ldots, A_{m}\right]$, where $A_{i}(p)$ is a column vector of $\mathbb{C}^{m}$ and $M$ is a Riemannian manifold with its Levi-Civita connection $\nabla$. Assume that, at $p_{0}, A\left(p_{0}\right)$ is a diagonal matrix $D=D\left(\lambda_{1}, \ldots, \lambda_{m}\right)$. Then, at $p_{0}$

$$
d(\operatorname{det} A)(Z)=\sum_{1 \leq j \leq m}\left(\prod_{k \neq j} \lambda_{k}\right) d A_{j}^{j}(Z),
$$

$\operatorname{Hess}(\operatorname{det} A)(Z, W)=\nabla d(\operatorname{det} A)(Z, W)$

$$
\begin{aligned}
= & \sum_{1 \leq j, k \leq m}\left(\prod_{s \neq j, k} \lambda_{s}\right) \operatorname{det}\left[\begin{array}{cc}
d A_{j}^{j}(Z) & d A_{j}^{k}(Z) \\
d A_{k}^{j}(W) & d A_{k}^{k}(W)
\end{array}\right] \\
& +\sum_{1 \leq j \leq m}\left(\prod_{s \neq j} \lambda_{s}\right) \operatorname{Hess} A_{j}^{j}(Z, W) .
\end{aligned}
$$

In particular, if $e_{1}, \ldots, e_{r}$ is an orthonormal basis of $T_{p_{0}} M$, then, at $p_{0}$,

$\triangle(\operatorname{det} A)=$ Trace Hess $(\operatorname{det} A)$

$$
\begin{aligned}
= & \sum_{1 \leq \alpha \leq r} \sum_{1 \leq j, k \leq m}\left(\prod_{s \neq j, k} \lambda_{s}\right) \operatorname{det}\left[\begin{array}{cc}
d A_{j}^{j}\left(e_{\alpha}\right) & d A_{j}^{k}\left(e_{\alpha}\right) \\
d A_{k}^{j}\left(e_{\alpha}\right) & d A_{k}^{k}\left(e_{\alpha}\right)
\end{array}\right] \\
& +\sum_{1 \leq j \leq m}\left(\prod_{s \neq j} \lambda_{s}\right) \triangle A_{j}^{j} .
\end{aligned}
$$

On each $\Omega_{2 k}^{0}$, the complex structure $J_{\omega}$ and the sub-vector bundle $\mathcal{K}_{\omega}^{\perp}$ are smooth. Moreover, $J_{\omega}$ is $g_{M}$-orthogonal. Thus, for each $p_{0} \in \Omega_{2 k}^{0}$, there exists a locally $g_{M}$-orthonormal frame of $\mathcal{K}_{\omega}^{\perp}$ defined on a neighbourhood of $p_{0}$, of the form $X_{1}, J_{\omega} X_{1}, \ldots, X_{k}, J_{\omega} X_{k}$. We enlarge this frame to a $g_{M^{-}}$ orthonormal local frame on $M$, on a neighbourhood of $p_{0}$ :

$$
X_{1}, Y_{1}=J_{\omega} X_{1}, \ldots, X_{k}, Y_{k}=J_{\omega} X_{k}, X_{k+1}, Y_{k+1}, \ldots, X_{n}, Y_{n}
$$

where $X_{k+1}, Y_{k+1}, \ldots X_{n}, Y_{n}$ is any $g_{M}$-orthonormal frame of $\mathcal{K}_{\omega}$, and which at $p_{0}$ is a diagonalizing basis of $F^{*} \omega$. Note that in general, without some restrictive conditions, it is not possible to get smooth diagonalizing $g_{M^{-}}$ orthonormal frames in a whole neighbourhood of a point $p_{0}$. We use the notations in Section 3.1. We define a local complex structure on a neighbourhood of $p_{0} \in \Omega_{2 k}^{0}$ as $\widetilde{J}=J_{\omega} \oplus J^{\prime}$, where $J_{\omega}$ is defined only on $\mathcal{K}_{\omega}^{\perp}$, and 
$J^{\prime}$ is the local complex structure on $\mathcal{K}_{\omega}$, defined on a neighbourhood of $p_{0}$ by

$$
J^{\prime} Z_{\alpha}=i Z_{\alpha}, \quad J^{\prime} Z_{\bar{\alpha}}=-i Z_{\bar{\alpha}}, \forall \alpha \geq k+1
$$

Thus, the vectors $Z_{\alpha}$ are of type $(1,0)$ with respect to $\widetilde{J}$, for $\forall \alpha$. Since $\widetilde{J}$ is $g_{M}$-orthogonal, then, $\forall \alpha, \beta$, on a neighbourhood of $p_{0}$,

$$
\left\langle\nabla_{Z} \widetilde{J}(\alpha), \beta\right\rangle=2 i\left\langle\nabla_{Z} \alpha, \beta\right\rangle=-\left\langle\alpha, \nabla_{Z} \widetilde{J}(\beta)\right\rangle, \quad\left\langle\nabla_{Z} \widetilde{J}(\alpha), \bar{\beta}\right\rangle=0 .
$$

Note that $F^{*} \omega$ and $\widetilde{g}$, where $\widetilde{g}$ is given in $(1.1)$, are both of type $(1,1)$ with respect to $\widetilde{J}$, and have the same kernel $\mathcal{K}_{\omega}$. They are related by $\widetilde{g}(X, Y)=F^{*} \omega\left(X, J_{\omega}(Y)\right)$. Set $\widetilde{g}_{A B}=\widetilde{g}(A, B)$, and define $\overline{\bar{B}}=B, \forall A, B \in$ $\{1, \ldots, n, \overline{1}, \ldots, \bar{n}\}$, and set $\epsilon_{\alpha}=+1, \epsilon_{\bar{\alpha}}=-1, \forall 1 \leq \alpha \leq n$. Let $\forall 1 \leq \alpha, \beta \leq$ $n$ and $A, B \in\{1, \ldots, n, \overline{1}, \ldots, \bar{n}\}, C \in\{1, \ldots, n\} \cup\{\overline{k+1}, \ldots, \bar{n}\}$. Then

$$
\begin{cases}F^{*} \omega(\alpha, C)=g(J d F(\alpha), d F(C))=0 & \forall p \text { near } p_{0} \\ F^{*} \omega(\alpha, \bar{\beta})=g(J d F(\alpha), d F(\bar{\beta}))=\frac{i}{2} \delta_{\alpha \beta} \cos \theta_{\alpha} & \text { at } p_{0} \\ \widetilde{g}_{A B}=i \epsilon_{B} F^{*} \omega(A, B)=i \epsilon_{B} g(J d F(A), d F(B)) & \forall p \text { near } p_{0} \\ \widetilde{g}_{\alpha C}=\widetilde{g}_{\bar{\alpha} \bar{C}}=0 & \forall p \text { near } p_{0} \\ \widetilde{g}_{\alpha \bar{\beta}}=\widetilde{g}_{\bar{\alpha} \beta}=\frac{1}{2} \delta_{\alpha \beta} \cos \theta_{\alpha} & \text { at } p_{0} .\end{cases}
$$

At a point $p_{0}$, with Kähler angles $\theta_{\alpha}, g_{M} \pm \widetilde{g}$ is represented in the unitary basis $\{\sqrt{2} \alpha, \sqrt{2} \bar{\alpha}\}$, by the diagonal matrix $g_{M} \pm \widetilde{g}=D\left(1 \pm \cos \theta_{1}, \ldots, 1 \pm\right.$ $\left.\cos \theta_{n}, 1 \pm \cos \theta_{1}, \ldots, 1 \pm \cos \theta_{n}\right)$, and so

$$
\operatorname{det}\left(g_{M} \pm \widetilde{g}\right)=\prod_{1 \leq \alpha \leq n}\left(1 \pm \cos \theta_{\alpha}\right)^{2} .
$$

If $p_{0}$ is a point without complex directions, $\cos \theta_{\alpha} \neq 1, \forall \alpha \in\{1, \ldots, n\}$, then $\tilde{g}<g_{M}$. Thus, on a neighbourhood of $p_{0}$, we may consider the map $\kappa$.

$$
\kappa=\frac{1}{2} \log \left(\frac{\operatorname{det}\left(g_{M}+\widetilde{g}\right)}{\operatorname{det}\left(g_{M}-\widetilde{g}\right)}\right)=\sum_{1 \leq \alpha \leq n} \log \left(\frac{1+\cos \theta_{\alpha}}{1-\cos \theta_{\alpha}}\right) .
$$

This map is continuous away from the complex directions, and smooth on each $\Omega_{2 k}^{0}$. We wish to compute $\triangle \kappa$ on $\Omega_{2 k}^{0}$.

Lemma 5.2. At $p_{0} \in \Omega_{2 k}^{0}$, without complex directions and for $Z, W \in$ $T_{p_{0}} M$,

$$
d\left(\operatorname{det}\left(g_{M} \pm \widetilde{g}\right)\right)(Z)= \pm 4 \sum_{1 \leq \mu \leq n} \frac{\prod_{1 \leq \alpha \leq n}\left(1 \pm \cos \theta_{\alpha}\right)^{2}}{\left(1 \pm \cos \theta_{\mu}\right)} d \widetilde{g}_{\mu \bar{\mu}}(Z)
$$




$$
\begin{aligned}
& \operatorname{Hess}\left(\operatorname{det}\left(g_{M} \pm \widetilde{g}\right)\right)(Z, W) \\
& =16\left(\prod_{1 \leq \alpha \leq n}\left(1 \pm \cos \theta_{\alpha}\right)^{2}\right) \sum_{\mu, \rho} \frac{1}{\left(1 \pm \cos \theta_{\mu}\right)\left(1 \pm \cos \theta_{\rho}\right)} d \widetilde{g}_{\mu \bar{\mu}}(Z) d \widetilde{g}_{\rho \bar{\rho}}(W) \\
& \quad-8\left(\prod_{1 \leq \alpha \leq n}\left(1 \pm \cos \theta_{\alpha}\right)^{2}\right) \sum_{\mu, \rho} \frac{1}{\left(1 \pm \cos \theta_{\mu}\right)\left(1 \pm \cos \theta_{\rho}\right)} d \widetilde{g}_{\mu \bar{\rho}}(W) d \widetilde{g}_{\rho \bar{\mu}}(Z) \\
& \pm 4\left(\prod_{1 \leq \alpha \leq n}\left(1 \pm \cos \theta_{\alpha}\right)^{2}\right) \sum_{\mu} \frac{1}{\left(1 \pm \cos \theta_{\mu}\right)} \operatorname{Hess} \widetilde{g}_{\mu \bar{\mu}}(Z, W) .
\end{aligned}
$$

Proof. Using the unitary basis $\{\sqrt{2} \alpha, \sqrt{2} \bar{\alpha}\}$ of $T_{p}^{c} M$, for $p$ near $p_{0}, g_{M} \pm \widetilde{g}$ is represented by the matrix

$$
\begin{aligned}
g_{M} \pm \widetilde{g} & =\left[\begin{array}{cc}
g_{M} \pm \widetilde{g}(\sqrt{2} \alpha, \sqrt{2} \bar{\gamma}) & g_{M} \pm \widetilde{g}(\sqrt{2} \alpha, \sqrt{2} \gamma) \\
g_{M} \pm \widetilde{g}(\sqrt{2} \bar{\alpha}, \sqrt{2} \bar{\gamma}) & g_{M} \pm \widetilde{g}(\sqrt{2} \bar{\alpha}, \sqrt{2} \gamma)
\end{array}\right] \\
& =\left[\begin{array}{cc}
\delta_{\alpha \gamma} \pm 2 \widetilde{g}_{\alpha \bar{\gamma}} & 0 \\
0 & \delta_{\alpha \gamma} \pm 2 \widetilde{g}_{\bar{\alpha} \gamma}
\end{array}\right]
\end{aligned}
$$

that at $p_{0}$ is the diagonal matrix $D\left(1 \pm \cos \theta_{1}, \ldots, 1 \pm \cos \theta_{n}, 1 \pm \cos \theta_{1}, \ldots, 1 \pm\right.$ $\left.\cos \theta_{n}\right)$. The lemma follows as a simple application of Lemma 5.1, and noting that $\widetilde{g}_{\mu \bar{\rho}}=\widetilde{g}_{\bar{\rho} \mu}$.

On $\Omega_{2 k}^{0}$,

$$
\begin{aligned}
2 \triangle \kappa= & \triangle \log \left(\operatorname{det}\left(g_{M}+\widetilde{g}\right)\right)-\triangle \log \left(\operatorname{det}\left(g_{M}-\widetilde{g}\right)\right) \\
= & \frac{\triangle\left(\operatorname{det}\left(g_{M}+\widetilde{g}\right)\right)}{\operatorname{det}\left(g_{M}+\widetilde{g}\right)}-\frac{\left\|d\left(\operatorname{det}\left(g_{M}+\widetilde{g}\right)\right)\right\|^{2}}{\left(\operatorname{det}\left(g_{M}+\widetilde{g}\right)\right)^{2}} \\
& -\frac{\triangle\left(\operatorname{det}\left(g_{M}-\widetilde{g}\right)\right)}{\operatorname{det}\left(g_{M}-\widetilde{g}\right)}+\frac{\left\|d\left(\operatorname{det}\left(g_{M}-\widetilde{g}\right)\right)\right\|^{2}}{\left(\operatorname{det}\left(g_{M}-\widetilde{g}\right)\right)^{2}} .
\end{aligned}
$$

From the above lemma and

$$
\begin{aligned}
\left\|d\left(\operatorname{det}\left(g_{M} \pm \widetilde{g}\right)\right)\right\|^{2} & =4 \sum_{\beta} d\left(\operatorname{det}\left(g_{M} \pm \widetilde{g}\right)\right)(\beta) d\left(\operatorname{det}\left(g_{M} \pm \widetilde{g}\right)\right)(\bar{\beta}) \\
\triangle \operatorname{det}\left(g_{M} \pm \widetilde{g}\right) & =4 \sum_{\beta} \operatorname{Hess}\left(\operatorname{det}\left(g_{M} \pm \widetilde{g}\right)\right)(\beta, \bar{\beta})
\end{aligned}
$$

we have at $p_{0}$,

$$
2 \triangle \kappa=\sum_{\beta, \mu, \rho} \frac{64\left(\cos \theta_{\mu}+\cos \theta_{\rho}\right)}{\sin ^{2} \theta_{\mu} \sin ^{2} \theta_{\rho}} d \widetilde{g}_{\mu \bar{\rho}}(\bar{\beta}) d \widetilde{g}_{\rho \bar{\mu}}(\beta)+\sum_{\beta, \mu} \frac{32}{\sin ^{2} \theta_{\mu}} \operatorname{Hess} \widetilde{g}_{\mu \bar{\mu}}(\beta, \bar{\beta}) .
$$

Recalling (2.4), and $d\left(F^{*} \omega(X, Y)\right)(Z)=\nabla_{Z} F^{*} \omega(X, Y)+F^{*} \omega\left(\nabla_{Z} X, Y\right)+$ $F^{*} \omega\left(X, \nabla_{Z} Y\right)$, using (5.4), we obtain: 
Lemma 5.3. $\forall p$ near $p_{0} \in \Omega_{2 k}^{0}, Z \in T_{p}^{c} M$, and $\mu, \gamma \in\{1, \ldots, n\}$

$$
\begin{gathered}
d \widetilde{g}_{\mu \bar{\gamma}}(Z)=i g_{Z} \mu \bar{\gamma}-i g_{Z} \bar{\gamma} \mu+2 \sum_{\rho}\left(\left\langle\nabla_{Z} \mu, \bar{\rho}\right\rangle \widetilde{g}_{\rho \bar{\gamma}}+\left\langle\nabla_{Z} \bar{\gamma}, \rho\right\rangle \widetilde{g}_{\mu \bar{\rho}}\right) \\
0=d \widetilde{g}_{\mu \gamma}(Z)=-i g_{Z} \mu \gamma+i g_{Z} \gamma \mu+2 \sum_{\rho}\left(\left\langle\nabla_{Z} \mu, \rho\right\rangle \widetilde{g}_{\bar{\rho} \gamma}-\left\langle\nabla_{Z} \gamma, \rho\right\rangle \widetilde{g}_{\mu \bar{\rho}}\right) .
\end{gathered}
$$

In particular, at $p_{0}$

$$
\begin{aligned}
d \widetilde{g}_{\mu \bar{\gamma}}(Z) & =i g_{Z} \mu \bar{\gamma}-i g_{Z} \bar{\gamma} \mu-\left(\cos \theta_{\mu}-\cos \theta_{\gamma}\right)\left\langle\nabla_{Z} \mu, \bar{\gamma}\right\rangle \\
0=d \widetilde{g}_{\mu \gamma}(Z) & =-i g_{Z} \mu \gamma+i g_{Z} \gamma \mu+\left(\cos \theta_{\mu}+\cos \theta_{\gamma}\right)\left\langle\nabla_{Z} \mu, \gamma\right\rangle .
\end{aligned}
$$

Lemma 5.4. If $F$ is minimal and $p_{0} \in \Omega_{2 k}^{0}$ is a point without complex directions, then for each $\mu \in\{1, \ldots, n\}$

$$
\begin{aligned}
& \sum_{1 \leq \beta \leq n} \operatorname{Hess} \widetilde{g}_{\mu \bar{\mu}}(\beta, \bar{\beta})=\sum_{1 \leq \beta \leq n} d\left(d \widetilde{g}_{\mu \bar{\mu}}(\beta)\right)(\bar{\beta})-d \widetilde{g}_{\mu \bar{\mu}}\left(\nabla_{\bar{\beta}} \beta\right) \\
& =\sum_{1 \leq \beta \leq n} i R^{N}\left(d F(\beta), d F(\bar{\beta}), d F(\mu), J d F(\bar{\mu})+i \cos \theta_{\mu} d F(\bar{\mu})\right) \\
& +2 \operatorname{Im}\left(R^{N}\left(d F(\beta), d F(\mu), d F(\bar{\beta}), J d F(\bar{\mu})+i \cos \theta_{\mu} d F(\bar{\mu})\right)\right) \\
& +2 \sum_{1 \leq \rho \leq n} \frac{\left(\cos \theta_{\rho}-\cos \theta_{\mu}\right)}{\sin ^{2} \theta_{\rho}}\left(\left|g_{\beta} \mu \rho\right|^{2}+\left|g_{\beta} \overline{\mu \rho}\right|^{2}\right) \\
& -2 \sum_{1 \leq \rho \leq n} \frac{\left(\cos \theta_{\rho}+\cos \theta_{\mu}\right)}{\sin ^{2} \theta_{\rho}}\left(\left|g_{\beta} \mu \bar{\rho}\right|^{2}+\left|g_{\beta} \bar{\mu} \rho\right|^{2}\right) \\
& +\sum_{1 \leq \rho \leq n}-2 i\left\langle\nabla_{\mu} \beta, \bar{\rho}\right\rangle g_{\bar{\beta}} \rho \bar{\mu}-2 i\left\langle\nabla_{\mu} \beta, \rho\right\rangle g_{\bar{\beta}} \overline{\rho \mu}-2 i\left\langle\nabla_{\mu} \bar{\beta}, \bar{\rho}\right\rangle g_{\rho} \beta \bar{\mu} \\
& +\sum_{1 \leq \rho \leq n} 2 i\left\langle\nabla_{\bar{\beta}} \mu, \bar{\rho}\right\rangle g_{\beta} \rho \bar{\mu}-2 i\left\langle\nabla_{\mu} \bar{\beta}, \rho\right\rangle g_{\bar{\rho}} \beta \bar{\mu}+2 i\left\langle\nabla_{\bar{\beta}} \mu, \rho\right\rangle g_{\bar{\rho}} \beta \bar{\mu} \\
& +\sum_{1 \leq \rho \leq n} 2 i\left\langle\nabla_{\bar{\mu}} \beta, \bar{\rho}\right\rangle g_{\bar{\beta}} \rho \mu+2 i\left\langle\nabla_{\bar{\mu}} \beta, \rho\right\rangle g_{\bar{\beta}} \bar{\rho} \mu+2 i\left\langle\nabla_{\bar{\mu}} \bar{\beta}, \bar{\rho}\right\rangle g_{\rho} \beta \mu \\
& +\sum_{1 \leq \rho \leq n}-2 i\left\langle\nabla_{\bar{\beta}} \bar{\mu}, \bar{\rho}\right\rangle g_{\rho} \beta \mu+2 i\left\langle\nabla_{\bar{\mu}} \bar{\beta}, \rho\right\rangle g_{\bar{\rho}} \beta \mu-2 i\left\langle\nabla_{\bar{\beta}} \bar{\mu}, \rho\right\rangle g_{\bar{\rho}} \beta \mu \\
& +\sum_{1 \leq \rho \leq n} 2 i\left\langle\nabla_{\bar{\beta}} \bar{\mu}, \bar{\rho}\right\rangle g_{\beta} \mu \rho+2 i\left\langle\nabla_{\bar{\beta}} \bar{\mu}, \rho\right\rangle g_{\beta} \mu \bar{\rho}-2 i\left\langle\nabla_{\bar{\beta}} \mu, \rho\right\rangle g_{\beta} \overline{\mu \rho} \\
& +\sum_{1 \leq \rho \leq n}-2 i\left\langle\nabla_{\bar{\beta}} \mu, \bar{\rho}\right\rangle g_{\beta} \bar{\mu} \rho+2 i\left\langle\nabla_{\beta} \mu, \bar{\rho}\right\rangle g_{\bar{\beta}} \rho \bar{\mu}-2 i\left\langle\nabla_{\beta} \mu, \bar{\rho}\right\rangle g_{\bar{\beta}} \bar{\mu} \rho \\
& +\sum_{1 \leq \rho \leq n} 2 i\left\langle\nabla_{\beta} \bar{\mu}, \rho\right\rangle g_{\bar{\beta}} \mu \bar{\rho}-2 i\left\langle\nabla_{\beta} \bar{\mu}, \rho\right\rangle g_{\bar{\beta}} \bar{\rho} \mu \\
& -2 \sum_{1 \leq \rho \leq n}\left(\cos \theta_{\mu}-\cos \theta_{\rho}\right)\left(\left|\left\langle\nabla_{\beta} \mu, \bar{\rho}\right\rangle\right|^{2}+\left|\left\langle\nabla_{\bar{\beta}} \mu, \bar{\rho}\right\rangle\right|^{2}\right) .
\end{aligned}
$$


Proof. We denote by $\nabla_{X} \nabla_{Y} d F$ the covariant derivative of $\nabla_{Y} d F$ in $T^{*} M \otimes$ $F^{-1} T N$, and by $\bar{R}(X, Y) \xi$, the curvature tensor of this connection, namely $(\bar{R}(X, Y) \xi)(Z)=R^{N}(d F(X), d F(Y)) \xi(Z)-\xi\left(R^{M}(X, Y) Z\right)$. From Lemma 5.3 , for $p$ on a neighbourhood of $p_{0}$,

$$
\begin{aligned}
d \widetilde{g}_{\mu \bar{\mu}}(\beta)= & i g\left(\nabla_{\beta} d F(\mu), J d F(\bar{\mu})\right)-i g\left(\nabla_{\beta} d F(\bar{\mu}), J d F(\mu)\right) \\
& +2 \sum_{\rho}\left(\left\langle\nabla_{\beta} \mu, \bar{\rho}\right\rangle \widetilde{g}_{\rho \bar{\mu}}+\left\langle\nabla_{\beta} \bar{\mu}, \rho\right\rangle \widetilde{g}_{\mu \bar{\rho}}\right) .
\end{aligned}
$$

Then at $p_{0}$,

$$
\begin{aligned}
& d\left(d \widetilde{g}_{\mu \bar{\mu}}(\beta)\right)(\bar{\beta}) \\
& =i g\left(\nabla_{\bar{\beta}}\left(\nabla_{\beta} d F(\mu)\right), J d F(\bar{\mu})\right)+i g\left(\nabla_{\beta} d F(\mu), \nabla_{\bar{\beta}}(J d F(\bar{\mu}))\right) \\
& -i g\left(\nabla_{\bar{\beta}}\left(\nabla_{\beta} d F(\bar{\mu})\right), J d F(\mu)\right)-i g\left(\nabla_{\beta} d F(\bar{\mu}), \nabla_{\bar{\beta}}(J d F(\mu))\right) \\
& +2 \sum_{\rho}\left(\nabla_{\bar{\beta}}\left(\left\langle\nabla_{\beta} \mu, \bar{\rho}\right\rangle\right) \widetilde{g}_{\rho \bar{\mu}}+\nabla_{\bar{\beta}}\left(\left\langle\nabla_{\beta} \bar{\mu}, \rho\right\rangle\right) \widetilde{g}_{\mu \bar{\rho}}\right) \\
& +\sum_{\rho} 2\left\langle\nabla_{\beta} \mu, \bar{\rho}\right\rangle d \widetilde{g}_{\rho \bar{\mu}}(\bar{\beta})+2\left\langle\nabla_{\beta} \bar{\mu}, \rho\right\rangle d \widetilde{g}_{\mu \bar{\rho}}(\bar{\beta}) \\
& =i g\left(\nabla_{\bar{\beta}}\left(\nabla_{\beta} d F(\mu)\right), J d F(\bar{\mu})\right)+i g\left(\nabla_{\beta} d F(\mu), J \nabla_{\bar{\beta}} d F(\bar{\mu})\right) \\
& +i g\left(\nabla_{\beta} d F(\mu), J d F\left(\nabla_{\bar{\beta}} \bar{\mu}\right)\right)-i g\left(\nabla_{\bar{\beta}}\left(\nabla_{\beta} d F(\bar{\mu})\right), J d F(\mu)\right) \\
& -i g\left(\nabla_{\beta} d F(\bar{\mu}), J \nabla_{\bar{\beta}} d F(\mu)\right)-i g\left(\nabla_{\beta} d F(\bar{\mu}), J d F\left(\nabla_{\bar{\beta}} \mu\right)\right) \\
& +\cos \theta_{\mu}\left(\nabla_{\bar{\beta}}\left(\left\langle\nabla_{\beta} \mu, \bar{\mu}\right\rangle\right)+\nabla_{\bar{\beta}}\left(\left\langle\mu, \nabla_{\beta} \bar{\mu}\right\rangle\right)\right)+(5.8) \\
& =i g\left(\nabla_{\bar{\beta}}\left(\nabla_{\beta} d F(\mu)\right), J d F(\bar{\mu})\right) \\
& +i g\left(\nabla_{\beta} d F(\mu), J \nabla_{\bar{\beta}} d F(\bar{\mu})\right) \\
& +\sum_{\rho} 2 i\left\langle\nabla_{\bar{\beta}} \bar{\mu}, \rho\right\rangle g_{\beta} \mu \bar{\rho}+2 i\left\langle\nabla_{\bar{\beta}} \bar{\mu}, \bar{\rho}\right\rangle g_{\beta} \mu \rho \\
& -i g\left(\nabla_{\bar{\beta}}\left(\nabla_{\beta} d F(\bar{\mu})\right), J d F(\mu)\right) \\
& -i g\left(\nabla_{\beta} d F(\bar{\mu}), J \nabla_{\bar{\beta}} d F(\mu)\right) \\
& +\sum_{\rho}-2 i\left\langle\nabla_{\bar{\beta}} \mu, \rho\right\rangle g_{\beta} \overline{\mu \rho}-2 i\left\langle\nabla_{\bar{\beta}} \mu, \bar{\rho}\right\rangle g_{\beta} \bar{\mu} \rho \\
& +\cos \theta_{\mu}\left(\nabla_{\bar{\beta}}\left(\left\langle\nabla_{\beta} \mu, \bar{\mu}\right\rangle\right)+\nabla_{\bar{\beta}}\left(\left\langle\mu, \nabla_{\beta} \bar{\mu}\right\rangle\right)\right) \\
& +(5.8) \text {. }
\end{aligned}
$$


The term (5.11) vanishes because $\left\langle\nabla_{\beta} \mu, \bar{\mu}\right\rangle=-\left\langle\mu, \nabla_{\beta} \bar{\mu}\right\rangle$ on a neighbourhood of $p_{0}$. Minimality of $F$ implies

$$
\begin{aligned}
\sum_{\beta} \nabla_{\bar{\beta}}\left(\nabla_{\beta} d F(\mu)\right) & =\sum_{\beta} \nabla_{\bar{\beta}}\left(\nabla_{\mu} d F(\beta)\right)=\sum_{\beta} \nabla_{\bar{\beta}} \nabla_{\mu} d F(\beta)+\nabla_{\mu} d F\left(\nabla_{\bar{\beta}} \beta\right) \\
= & \sum_{\beta} \nabla_{\mu} \nabla_{\bar{\beta}} d F(\beta)-\nabla_{[\mu, \bar{\beta}]} d F(\beta)+(\bar{R}(\mu, \bar{\beta}) d F)(\beta)+\nabla_{\mu} d F\left(\nabla_{\bar{\beta}} \beta\right) \\
= & \sum_{\beta} \nabla_{\mu}\left(\nabla_{\bar{\beta}} d F(\beta)\right)-\nabla_{\bar{\beta}} d F\left(\nabla_{\mu} \beta\right)-\nabla_{[\mu, \bar{\beta}]} d F(\beta) \\
& +R^{N}(d F(\mu), d F(\bar{\beta})) d F(\beta)-d F\left(R^{M}(\mu, \bar{\beta}) \beta\right)+\nabla_{\mu} d F\left(\nabla_{\bar{\beta}} \beta\right) \\
= & \sum_{\beta} \sum_{\rho}-2\left\langle\nabla_{\mu} \beta, \bar{\rho}\right\rangle \nabla_{\bar{\beta}} d F(\rho)+\sum_{\rho}-2\left\langle\nabla_{\mu} \beta, \rho\right\rangle \nabla_{\bar{\beta}} d F(\bar{\rho}) \\
& -\sum_{\rho}\left(2\left\langle\nabla_{\mu} \bar{\beta}, \bar{\rho}\right\rangle-2\left\langle\nabla_{\bar{\beta}} \mu, \bar{\rho}\right\rangle\right) \nabla_{\rho} d F(\beta) \\
& -\sum_{\rho}\left(2\left\langle\nabla_{\mu} \bar{\beta}, \rho\right\rangle-2\left\langle\nabla_{\bar{\beta}} \mu, \rho\right\rangle\right) \nabla_{\bar{\rho}} d F(\beta) \\
& +R^{N}(d F(\mu), d F(\bar{\beta})) d F(\beta)-d F\left(R^{M}(\mu, \bar{\beta}) \beta\right) \\
& +\sum_{\rho} 2\left\langle\nabla_{\bar{\beta}} \beta, \bar{\rho}\right\rangle \nabla_{\mu} d F(\rho)+\sum_{\rho} 2\left\langle\nabla_{\bar{\beta}} \beta, \rho\right\rangle \nabla_{\mu} d F(\bar{\rho}) .
\end{aligned}
$$

Hence

$$
\begin{aligned}
\sum_{\beta}(5.9)= & \sum_{\beta} i R^{N}(d F(\mu), d F(\bar{\beta}), d F(\beta), J d F(\bar{\mu}))-\cos \theta_{\mu} R^{M}(\mu, \bar{\beta}, \beta, \bar{\mu}) \\
& +\sum_{\beta \rho}-2 i\left\langle\nabla_{\mu} \beta, \bar{\rho}\right\rangle g_{\bar{\beta}} \rho \bar{\mu}-2 i\left\langle\nabla_{\mu} \beta, \rho\right\rangle g_{\bar{\beta}} \overline{\rho \mu} \\
& +\sum_{\beta \rho} 2 i\left(-\left\langle\nabla_{\mu} \bar{\beta}, \bar{\rho}\right\rangle+\left\langle\nabla_{\bar{\beta}} \mu, \bar{\rho}\right\rangle\right) g_{\rho} \beta \bar{\mu} \\
& +\sum_{\beta \rho} 2 i\left(-\left\langle\nabla_{\mu} \bar{\beta}, \rho\right\rangle+\left\langle\nabla_{\bar{\beta}} \mu, \rho\right\rangle\right) g_{\bar{\rho}} \beta \bar{\mu} \\
& +\sum_{\beta \rho} 2 i\left\langle\nabla_{\bar{\beta}} \beta, \bar{\rho}\right\rangle g_{\mu} \rho \bar{\mu}+2 i\left\langle\nabla_{\bar{\beta}} \beta, \rho\right\rangle g_{\mu} \overline{\rho \mu}
\end{aligned}
$$

Similarly 


$$
\begin{aligned}
-\sum_{\beta}(5.10)= & \sum_{\beta} i R^{N}(d F(\bar{\mu}), d F(\bar{\beta}), d F(\beta), J d F(\mu))+\cos \theta_{\mu} R^{M}(\bar{\mu}, \bar{\beta}, \beta, \mu) \\
& +\sum_{\beta \rho}-2 i\left\langle\nabla_{\bar{\mu}} \beta, \bar{\rho}\right\rangle g_{\bar{\beta}} \rho \mu-2 i\left\langle\nabla_{\bar{\mu}} \beta, \rho\right\rangle g_{\bar{\beta}} \bar{\rho} \mu \\
& +\sum_{\beta \rho} 2 i\left(-\left\langle\nabla_{\bar{\mu}} \bar{\beta}, \bar{\rho}\right\rangle+\left\langle\nabla_{\bar{\beta}} \bar{\mu}, \bar{\rho}\right\rangle\right) g_{\rho} \beta \mu \\
& +\sum_{\beta \rho} 2 i\left(-\left\langle\nabla_{\bar{\mu}} \bar{\beta}, \rho\right\rangle+\left\langle\nabla_{\bar{\beta}} \bar{\mu}, \rho\right\rangle\right) g_{\bar{\rho}} \beta \mu \\
& +\sum_{\beta \rho} 2 i\left\langle\nabla_{\bar{\beta}} \beta, \bar{\rho}\right\rangle g_{\bar{\mu}} \rho \mu+2 i\left\langle\nabla_{\bar{\beta}} \beta, \rho\right\rangle g_{\bar{\mu}} \bar{\rho} \mu .
\end{aligned}
$$

Using Bianchi identity,

$$
\begin{aligned}
i R^{N} & (d F(\mu), d F(\bar{\beta}), d F(\beta), J d F(\bar{\mu})) \\
& -i R^{N}(d F(\bar{\mu}), d F(\bar{\beta}), d F(\beta), J d F(\mu)) \\
= & -i R^{N}(d F(\beta), d F(\mu), d F(\bar{\beta}), J d F(\bar{\mu})) \\
& -i R^{N}(d F(\bar{\beta}), d F(\beta), d F(\mu), J d F(\bar{\mu})) \\
& -i R^{N}(d F(\bar{\mu}), d F(\bar{\beta}), d F(\beta), J d F(\mu)) \\
= & i R^{N}(d F(\beta), d F(\bar{\beta}), d F(\mu), J d F(\bar{\mu})) \\
& +2 \operatorname{Im}\left(R^{N}(d F(\beta), d F(\mu), d F(\bar{\beta}), J d F(\bar{\mu}))\right),
\end{aligned}
$$

and by Gauss equation, and minimality of $F$,

$$
\begin{aligned}
& \sum_{\beta}-R^{M}(\mu, \bar{\beta}, \beta, \bar{\mu})-R^{M}(\bar{\mu}, \bar{\beta}, \beta, \mu) \\
& =\sum_{\beta} R^{M}(\beta, \mu, \bar{\beta}, \bar{\mu})+R^{M}(\bar{\beta}, \beta, \mu, \bar{\mu})-R^{M}(\bar{\mu}, \bar{\beta}, \beta, \mu) \\
& =\sum_{\beta}-R^{M}(\beta, \bar{\beta}, \mu, \bar{\mu})+2 R^{M}(\beta, \mu, \bar{\beta}, \bar{\mu}) \\
& =\sum_{\beta}-R^{N}(d F(\beta), d F(\bar{\beta}), d F(\mu), d F(\bar{\mu})) \\
& \quad-g\left(\nabla_{\beta} d F(\mu), \nabla_{\bar{\beta}} d F(\bar{\mu})\right)+g\left(\nabla_{\beta} d F(\bar{\mu}), \nabla_{\bar{\beta}} d F(\mu)\right) \\
& \quad+2 R^{N}(d F(\beta), d F(\mu), d F(\bar{\beta}), d F(\bar{\mu})) \\
& \quad+2 g\left(\nabla_{\beta} d F(\bar{\beta}), \nabla_{\mu} d F(\bar{\mu})\right)-2 g\left(\nabla_{\beta} d F(\bar{\mu}), \nabla_{\mu} d F(\bar{\beta})\right)
\end{aligned}
$$




$$
\begin{aligned}
= & \sum_{\beta}-R^{N}(d F(\beta), d F(\bar{\beta}), d F(\mu), d F(\bar{\mu})) \\
& +2 R^{N}(d F(\beta), d F(\mu), d F(\bar{\beta}), d F(\bar{\mu})) \\
& -g\left(\nabla_{\beta} d F(\mu), \nabla_{\bar{\beta}} d F(\bar{\mu})\right)-g\left(\nabla_{\beta} d F(\bar{\mu}), \nabla_{\mu} d F(\bar{\beta})\right) .
\end{aligned}
$$

Note that $R^{N}(d F(\beta), d F(\mu), d F(\bar{\beta}), d F(\bar{\mu}))=\operatorname{Im}\left(i R^{N}(d F(\beta), d F(\mu)\right.$, $d F(\bar{\beta}), d F(\bar{\mu})))$, since it is real. Therefore,

$$
\begin{aligned}
& \sum_{\beta} d\left(d \widetilde{g}_{\mu \bar{\mu}}(\beta)\right)(\bar{\beta}) \\
& =\sum_{\beta} i R^{N}\left(d F(\beta), d F(\bar{\beta}), d F(\mu), J d F(\bar{\mu})+i \cos \theta_{\mu} d F(\bar{\mu})\right) \\
& +2 \operatorname{Im}\left(R^{N}\left(d F(\beta), d F(\mu), d F(\bar{\beta}), J d F(\bar{\mu})+i \cos \theta_{\mu} d F(\bar{\mu})\right)\right) \\
& -\cos \theta_{\mu} g\left(\nabla_{\beta} d F(\mu), \nabla_{\bar{\beta}} d F(\bar{\mu})\right)-\cos \theta_{\mu} g\left(\nabla_{\beta} d F(\bar{\mu}), \nabla_{\mu} d F(\bar{\beta})\right) \\
& +\sum_{\rho}-2 i\left\langle\nabla_{\mu} \beta, \bar{\rho}\right\rangle g_{\bar{\beta}} \rho \bar{\mu}-2 i\left\langle\nabla_{\mu} \beta, \rho\right\rangle g_{\bar{\beta}} \overline{\rho \mu} \\
& +\sum_{\rho} 2 i\left(-\left\langle\nabla_{\mu} \bar{\beta}, \bar{\rho}\right\rangle+\left\langle\nabla_{\bar{\beta}} \mu, \bar{\rho}\right\rangle\right) g_{\rho} \beta \bar{\mu} \\
& +\sum_{\rho} 2 i\left(-\left\langle\nabla_{\mu} \bar{\beta}, \rho\right\rangle+\left\langle\nabla_{\bar{\beta}} \mu, \rho\right\rangle\right) g_{\bar{\rho}} \beta \bar{\mu} \\
& +\sum_{\rho} 2 i\left\langle\nabla_{\bar{\beta}} \beta, \bar{\rho}\right\rangle g_{\mu} \rho \bar{\mu}+2 i\left\langle\nabla_{\bar{\beta}} \beta, \rho\right\rangle g_{\mu} \overline{\rho \mu} \\
& +\sum_{\rho} 2 i\left\langle\nabla_{\bar{\mu}} \beta, \bar{\rho}\right\rangle g_{\bar{\beta}} \rho \mu+2 i\left\langle\nabla_{\bar{\mu}} \beta, \rho\right\rangle g_{\bar{\beta}} \bar{\rho} \mu \\
& +\sum_{\rho} 2 i\left(\left\langle\nabla_{\bar{\mu}} \bar{\beta}, \bar{\rho}\right\rangle-\left\langle\nabla_{\bar{\beta}} \bar{\mu}, \bar{\rho}\right\rangle\right) g_{\rho} \beta \mu+2 i\left(\left\langle\nabla_{\bar{\mu}} \bar{\beta}, \rho\right\rangle-\left\langle\nabla_{\bar{\beta}} \bar{\mu}, \rho\right\rangle\right) g_{\bar{\rho}} \beta \mu \\
& \text { (5.14) } \quad+\sum_{\rho}-2 i\left\langle\nabla_{\bar{\beta}} \beta, \bar{\rho}\right\rangle g_{\bar{\mu}} \rho \mu-2 i\left\langle\nabla_{\bar{\beta}} \beta, \rho\right\rangle g_{\bar{\mu}} \bar{\rho} \mu \\
& +i g\left(\nabla_{\beta} d F(\mu), J \nabla_{\bar{\beta}} d F(\bar{\mu})\right)-i g\left(\nabla_{\beta} d F(\bar{\mu}), J \nabla_{\bar{\beta}} d F(\mu)\right) \\
& +\sum_{\rho} 2 i\left\langle\nabla_{\bar{\beta}} \bar{\mu}, \rho\right\rangle g_{\beta} \mu \bar{\rho}+2 i\left\langle\nabla_{\bar{\beta}} \bar{\mu}, \bar{\rho}\right\rangle g_{\beta} \mu \rho \\
& \sum_{\rho}-2 i\left\langle\nabla_{\bar{\beta}} \mu, \rho\right\rangle g_{\beta} \overline{\mu \rho}-2 i\left\langle\nabla_{\bar{\beta}} \mu, \bar{\rho}\right\rangle g_{\beta} \bar{\mu} \rho+(5.8) .
\end{aligned}
$$

Using the unitary basis $\left\{\frac{\sqrt{2}}{\sin \theta_{\rho}} \Phi(\rho), \frac{\sqrt{2}}{\sin \theta_{\rho}} \Phi(\bar{\rho})\right\}$ of the normal bundle, and 


$$
\begin{aligned}
(5.12)+(5.15) & \\
= & -\sum_{\beta, \rho} \frac{2 \cos \theta_{\mu}}{\sin ^{2} \theta_{\rho}}\left(\left|g_{\beta} \mu \rho\right|^{2}+\left|g_{\beta} \mu \bar{\rho}\right|^{2}\right)-\sum_{\beta, \rho} \frac{2 \cos \theta_{\mu}}{\sin ^{2} \theta_{\rho}}\left(\left|g_{\beta} \bar{\mu} \rho\right|^{2}+\left|g_{\beta} \overline{\mu \rho}\right|^{2}\right) \\
& -\sum_{\beta, \rho} \frac{2 \cos \theta_{\rho}}{\sin ^{2} \theta_{\rho}}\left(\left|g_{\beta} \mu \bar{\rho}\right|^{2}-\left|g_{\beta} \mu \rho\right|^{2}\right)+\sum_{\beta, \rho} \frac{2 \cos \theta_{\rho}}{\sin ^{2} \theta_{\rho}}\left(\left|g_{\beta} \overline{\mu \rho}\right|^{2}-\left|g_{\beta} \bar{\mu} \rho\right|^{2}\right) \\
= & 2 \sum_{\beta, \rho} \frac{\left(\cos \theta_{\rho}-\cos \theta_{\mu}\right)}{\sin ^{2} \theta_{\rho}}\left|g_{\beta} \mu \rho\right|^{2}-2 \sum_{\beta, \rho} \frac{\left(\cos \theta_{\rho}+\cos \theta_{\mu}\right)}{\sin ^{2} \theta_{\rho}}\left|g_{\beta} \mu \bar{\rho}\right|^{2} \\
& -2 \sum_{\beta, \rho} \frac{\left(\cos \theta_{\rho}+\cos \theta_{\mu}\right)}{\sin ^{2} \theta_{\rho}}\left|g_{\beta} \bar{\mu} \rho\right|^{2}+2 \sum_{\beta, \rho} \frac{\left(\cos \theta_{\rho}-\cos \theta_{\mu}\right)}{\sin ^{2} \theta_{\rho}}\left|g_{\beta} \overline{\mu \rho}\right|^{2} .
\end{aligned}
$$

Applying Lemma 5.3 we have

$$
\begin{aligned}
d \widetilde{g}_{\mu \bar{\mu}}\left(\nabla_{\bar{\beta}} \beta\right)= & \sum_{\rho} 2\left\langle\nabla_{\bar{\beta}} \beta, \bar{\rho}\right\rangle d \widetilde{g}_{\mu \bar{\mu}}(\rho)+\sum_{\rho} 2\left\langle\nabla_{\bar{\beta}} \beta, \rho\right\rangle d \widetilde{g}_{\mu \bar{\mu}}(\bar{\rho}) \\
= & 2 i \sum_{\rho}\left(\left\langle\nabla_{\bar{\beta}} \beta, \bar{\rho}\right\rangle g_{\rho} \mu \bar{\mu}-\left\langle\nabla_{\bar{\beta}} \beta, \bar{\rho}\right\rangle g_{\rho} \bar{\mu} \mu\right. \\
& \left.+\left\langle\nabla_{\bar{\beta}} \beta, \rho\right\rangle g_{\bar{\rho}} \mu \bar{\mu}-\left\langle\nabla_{\bar{\beta}} \beta, \rho\right\rangle g_{\bar{\rho}} \bar{\mu} \mu\right) \\
= & (5.13)+(5.14) .
\end{aligned}
$$

Finally

$$
\begin{aligned}
(5.8)= & \sum_{\rho} 2\left\langle\nabla_{\beta} \mu, \bar{\rho}\right\rangle\left(i g_{\bar{\beta}} \rho \bar{\mu}-i g_{\bar{\beta}} \bar{\mu} \rho\right) \\
& -\sum_{\rho} 2\left\langle\nabla_{\beta} \mu, \bar{\rho}\right\rangle\left(\cos \theta_{\rho}-\cos \theta_{\mu}\right)\left\langle\nabla_{\bar{\beta}} \rho, \bar{\mu}\right\rangle \\
& +\sum_{\rho} 2\left\langle\nabla_{\beta} \bar{\mu}, \rho\right\rangle\left(i g_{\bar{\beta}} \mu \bar{\rho}-i g_{\bar{\beta}} \bar{\rho} \mu\right) \\
& -\sum_{\rho} 2\left\langle\nabla_{\beta} \bar{\mu}, \rho\right\rangle\left(\cos \theta_{\mu}-\cos \theta_{\rho}\right)\left\langle\nabla_{\bar{\beta}} \mu, \bar{\rho}\right\rangle \\
= & \sum_{\rho} 2 i\left\langle\nabla_{\beta} \mu, \bar{\rho}\right\rangle g_{\bar{\beta}} \rho \bar{\mu}-2 i\left\langle\nabla_{\beta} \mu, \bar{\rho}\right\rangle g_{\bar{\beta}} \bar{\mu} \rho \\
& +\sum_{\rho} 2 i\left\langle\nabla_{\beta} \bar{\mu}, \rho\right\rangle g_{\bar{\beta}} \mu \bar{\rho}-2 i\left\langle\nabla_{\beta} \bar{\mu}, \rho\right\rangle g_{\bar{\beta}} \bar{\rho} \mu \\
& -2 \sum_{\rho}\left(\cos \theta_{\mu}-\cos \theta_{\rho}\right)\left(\left|\left\langle\nabla_{\beta} \mu, \bar{\rho}\right\rangle\right|^{2}+\left|\left\langle\nabla_{\bar{\beta}} \mu, \bar{\rho}\right\rangle\right|^{2}\right) .
\end{aligned}
$$

These expressions lead to the expression of the lemma. 
Finally, we have:

Proposition 5.1. If $F$ is minimal without complex directions, then for each $0 \leq k \leq 2 n$ at each $p_{0} \in \Omega_{2 k}^{0}$,

$$
\begin{aligned}
\triangle \kappa= & 4 i \sum_{\beta} \operatorname{Ricci}^{N}(J d F(\beta), d F(\bar{\beta})) \\
& +\sum_{\beta, \mu} \frac{32}{\sin ^{2} \theta_{\mu}} \operatorname{Im}\left(R^{N}\left(d F(\beta), d F(\mu), d F(\bar{\beta}), J d F(\bar{\mu})+i \cos \theta_{\mu} d F(\bar{\mu})\right)\right) \\
& -\sum_{\beta, \mu, \rho} \frac{64\left(\cos \theta_{\mu}+\cos \theta_{\rho}\right)}{\sin ^{2} \theta_{\mu} \sin ^{2} \theta_{\rho}} \operatorname{Re}\left(g_{\beta} \mu \bar{\rho} g_{\bar{\beta}} \rho \bar{\mu}\right) \\
& +\sum_{\beta, \mu, \rho} \frac{32\left(\cos \theta_{\rho}-\cos \theta_{\mu}\right)}{\sin ^{2} \theta_{\mu} \sin ^{2} \theta_{\rho}}\left(\left|g_{\beta} \mu \rho\right|^{2}+\left|g_{\bar{\beta}} \mu \rho\right|^{2}\right) \\
& +\sum_{\beta, \mu, \rho} \frac{32\left(\cos \theta_{\mu}+\cos \theta_{\rho}\right)}{\sin ^{2} \theta_{\mu}}\left(\left|\left\langle\nabla_{\beta} \mu, \rho\right\rangle\right|^{2}+\left|\left\langle\nabla_{\bar{\beta}} \mu, \rho\right\rangle\right|^{2}\right) .
\end{aligned}
$$

Proof. From (5.7) and Lemma 5.4 we get

$2 \triangle \kappa$

$$
\begin{aligned}
= & \sum_{\beta, \mu, \rho} \frac{64\left(\cos \theta_{\mu}+\cos \theta_{\rho}\right)}{\sin ^{2} \theta_{\mu} \sin ^{2} \theta_{\rho}} d \widetilde{g}_{\mu \bar{\rho}}(\bar{\beta}) d \widetilde{g}_{\rho \bar{\mu}}(\beta) \\
& +\sum_{\beta, \mu} \frac{32 i}{\sin ^{2} \theta_{\mu}} R^{N}\left(d F(\beta), d F(\bar{\beta}), d F(\mu), J d F(\bar{\mu})+i \cos \theta_{\mu} d F(\bar{\mu})\right) \\
& +\sum_{\beta, \mu} \frac{64}{\sin ^{2} \theta_{\mu}} \operatorname{Im}\left(R^{N}(d F(\beta), d F(\mu), d F(\bar{\beta}), J d F(\bar{\mu})\right. \\
& \left.\left.+i \cos \theta_{\mu} d F(\bar{\mu})\right)\right) \\
& +\sum_{\beta, \mu, \rho} \frac{64\left(\cos \theta_{\rho}-\cos \theta_{\mu}\right)}{\sin ^{2} \theta_{\mu} \sin ^{2} \theta_{\rho}}\left(\left|g_{\beta} \mu \rho\right|^{2}+\left|g_{\beta} \overline{\mu \rho}\right|^{2}\right) \\
& -\sum_{\beta, \mu, \rho} \frac{64\left(\cos \theta_{\rho}+\cos \theta_{\mu}\right)}{\sin ^{2} \theta_{\mu} \sin ^{2} \theta_{\rho}}\left(\left|g_{\beta} \mu \bar{\rho}\right|^{2}+\left|g_{\beta} \bar{\mu} \rho\right|^{2}\right)
\end{aligned}
$$

$$
+\sum_{\beta, \mu, \rho}-\frac{64 i}{\sin ^{2} \theta_{\mu}}\left\langle\nabla_{\mu} \beta, \bar{\rho}\right\rangle g_{\bar{\beta}} \rho \bar{\mu}-\frac{64 i}{\sin ^{2} \theta_{\mu}}\left\langle\nabla_{\mu} \beta, \rho\right\rangle g_{\bar{\beta}} \overline{\rho \mu}-\frac{64 i}{\sin ^{2} \theta_{\mu}}\left\langle\nabla_{\mu} \bar{\beta}, \bar{\rho}\right\rangle g_{\rho} \beta \bar{\mu}
$$

$$
+\sum_{\beta, \mu, \rho} \frac{64 i}{\sin ^{2} \theta_{\mu}}\left\langle\nabla_{\bar{\beta}} \mu, \bar{\rho}\right\rangle g_{\beta} \rho \bar{\mu}-\frac{64 i}{\sin ^{2} \theta_{\mu}}\left\langle\nabla_{\mu} \bar{\beta}, \rho\right\rangle g_{\bar{\rho}} \beta \bar{\mu}+\frac{64 i}{\sin ^{2} \theta_{\mu}}\left\langle\nabla_{\bar{\beta}} \mu, \rho\right\rangle g_{\bar{\rho}} \beta \bar{\mu}
$$


(5.18)

$+\sum_{\beta, \mu, \rho} \frac{64 i}{\sin ^{2} \theta_{\mu}}\left\langle\nabla_{\bar{\mu}} \beta, \bar{\rho}\right\rangle g_{\bar{\beta}} \rho \mu+\frac{64 i}{\sin ^{2} \theta_{\mu}}\left\langle\nabla_{\bar{\mu}} \beta, \rho\right\rangle g_{\bar{\beta}} \bar{\rho} \mu+\frac{64 i}{\sin ^{2} \theta_{\mu}}\left\langle\nabla_{\bar{\mu}} \bar{\beta}, \bar{\rho}\right\rangle g_{\rho} \beta \mu$

$(5.19)$

$+\sum_{\beta, \mu, \rho}-\frac{64 i}{\sin ^{2} \theta_{\mu}}\left\langle\nabla_{\bar{\beta}} \bar{\mu}, \bar{\rho}\right\rangle g_{\rho} \beta \mu+\frac{64 i}{\sin ^{2} \theta_{\mu}}\left\langle\nabla_{\bar{\mu}} \bar{\beta}, \rho\right\rangle g_{\bar{\rho}} \beta \mu-\frac{64 i}{\sin ^{2} \theta_{\mu}}\left\langle\nabla_{\bar{\beta}} \bar{\mu}, \rho\right\rangle g_{\bar{\rho}} \beta \mu$

$(5.20)$

$+\sum_{\beta, \mu, \rho} \frac{64 i}{\sin ^{2} \theta_{\mu}}\left\langle\nabla_{\bar{\beta}} \bar{\mu}, \bar{\rho}\right\rangle g_{\beta} \mu \rho+\frac{64 i}{\sin ^{2} \theta_{\mu}}\left\langle\nabla_{\bar{\beta}} \bar{\mu}, \rho\right\rangle g_{\beta} \mu \bar{\rho}-\frac{64 i}{\sin ^{2} \theta_{\mu}}\left\langle\nabla_{\bar{\beta}} \mu, \rho\right\rangle g_{\beta} \overline{\mu \rho}$

$+\sum_{\beta, \mu, \rho}-\frac{64 i}{\sin ^{2} \theta_{\mu}}\left\langle\nabla_{\bar{\beta}} \mu, \bar{\rho}\right\rangle g_{\beta} \bar{\mu} \rho+\frac{64 i}{\sin ^{2} \theta_{\mu}}\left\langle\nabla_{\beta} \mu, \bar{\rho}\right\rangle g_{\bar{\beta}} \rho \bar{\mu}-\frac{64 i}{\sin ^{2} \theta_{\mu}}\left\langle\nabla_{\beta} \mu, \bar{\rho}\right\rangle g_{\bar{\beta}} \bar{\mu} \rho$

$(5.22)$

$$
\begin{aligned}
& +\sum_{\beta, \mu, \rho} \frac{64 i}{\sin ^{2} \theta_{\mu}}\left\langle\nabla_{\beta} \bar{\mu}, \rho\right\rangle g_{\bar{\beta}} \mu \bar{\rho}-\frac{64 i}{\sin ^{2} \theta_{\mu}}\left\langle\nabla_{\beta} \bar{\mu}, \rho\right\rangle g_{\bar{\beta}} \bar{\rho} \mu \\
& -\sum_{\beta, \mu, \rho} \frac{64\left(\cos \theta_{\mu}-\cos \theta_{\rho}\right)}{\sin ^{2} \theta_{\mu}}\left(\left|\left\langle\nabla_{\beta} \mu, \bar{\rho}\right\rangle\right|^{2}+\left|\left\langle\nabla_{\bar{\beta}} \mu, \bar{\rho}\right\rangle\right|^{2}\right) .
\end{aligned}
$$

Interchanging $\rho$ with $\beta$ in the first term of (5.16) (that we named by (5.16)(1), and similarly to other equations), we see that $(5.16)(1)+(5.17)(2)=0$. Interchanging $\rho$ with $\beta$ in (5.18)(1), we get (5.18)(1) $+(5.19)(2)=0$. In (5.16) (2), $\left\langle\nabla_{\mu} \beta, \rho\right\rangle$ is skew-symmetric on $\rho$ and $\beta$, and $g_{\bar{\beta}} \overline{\rho \mu}$ is symmetric on $\rho$ and $\beta$. Hence $(5.16)(2)=0$. Similarly $(5.16)(3)=(5.18)(2)=(5.18)(3)=0$. If we interchange $\rho$ with $\mu$ in (5.17)(1),

$$
(5.17)(1)+(5.20)(2)=-\sum_{\beta, \mu, \rho} \frac{64 i\left(\sin ^{2} \theta_{\mu}-\sin ^{2} \theta_{\rho}\right)}{\sin ^{2} \theta_{\mu} \sin ^{2} \theta_{\rho}}\left\langle\nabla_{\bar{\beta}} \bar{\mu}, \rho\right\rangle g_{\beta} \mu \bar{\rho} .
$$

Interchanging $\rho$ with $\mu$ in $(5.17)(3)$, we get

$$
(5.17)(3)+(5.20)(3)=-\sum_{\beta, \mu, \rho} \frac{64 i\left(\sin ^{2} \theta_{\mu}+\sin ^{2} \theta_{\rho}\right)}{\sin ^{2} \theta_{\mu} \sin ^{2} \theta_{\rho}}\left\langle\nabla_{\bar{\beta}} \mu, \rho\right\rangle g_{\beta} \overline{\mu \rho} .
$$

Interchanging $\rho$ with $\mu$ in (5.19)(1), we get

$$
(5.19)(1)+(5.20)(1)=\sum_{\beta, \mu, \rho} \frac{64 i\left(\sin ^{2} \theta_{\mu}+\sin ^{2} \theta_{\rho}\right)}{\sin ^{2} \theta_{\mu} \sin ^{2} \theta_{\rho}}\left\langle\nabla_{\bar{\beta}} \bar{\mu}, \bar{\rho}\right\rangle g_{\beta} \mu \rho .
$$

Interchanging $\rho$ with $\mu$ in (5.19)(3), we get

$$
(5.19)(3)+(5.21)(1)=\sum_{\beta, \mu, \rho} \frac{64 i\left(\sin ^{2} \theta_{\mu}-\sin ^{2} \theta_{\rho}\right)}{\sin ^{2} \theta_{\mu} \sin ^{2} \theta_{\rho}}\left\langle\nabla_{\bar{\beta}} \mu, \bar{\rho}\right\rangle g_{\beta} \bar{\mu} \rho .
$$


Interchanging $\rho$ with $\mu$ in $(5.21)(2)$,

$$
(5.21)(2)+(5.22)(1)=\sum_{\beta, \mu, \rho} \frac{64 i\left(-\sin ^{2} \theta_{\mu}+\sin ^{2} \theta_{\rho}\right)}{\sin ^{2} \theta_{\mu} \sin ^{2} \theta_{\rho}}\left\langle\nabla_{\beta} \bar{\mu}, \rho\right\rangle g_{\bar{\beta}} \mu \bar{\rho} .
$$

Interchanging $\rho$ with $\mu$ in $(5.22)(2)$, we obtain

$$
(5.22)(2)+(5.21)(3)=\sum_{\beta, \mu, \rho} \frac{64 i\left(\sin ^{2} \theta_{\mu}-\sin ^{2} \theta_{\rho}\right)}{\sin ^{2} \theta_{\mu} \sin ^{2} \theta_{\rho}}\left\langle\nabla_{\beta} \mu, \bar{\rho}\right\rangle g_{\bar{\beta}} \bar{\mu} \rho .
$$

Therefore,

$2 \triangle \kappa$

$$
\begin{aligned}
= & \sum_{\beta, \mu, \rho} \frac{64\left(\cos \theta_{\mu}+\cos \theta_{\rho}\right)}{\sin ^{2} \theta_{\mu} \sin ^{2} \theta_{\rho}} d \widetilde{g}_{\mu \bar{\rho}}(\bar{\beta}) d \widetilde{g}_{\rho \bar{\mu}}(\beta) \\
& +\sum_{\beta, \mu} \frac{32 i}{\sin ^{2} \theta_{\mu}} R^{N}\left(d F(\beta), d F(\bar{\beta}), d F(\mu), J d F(\bar{\mu})+i \cos \theta_{\mu} d F(\bar{\mu})\right) \\
& +\sum_{\beta, \mu} \frac{64}{\sin ^{2} \theta_{\mu}} \operatorname{Im}\left(R^{N}(d F(\beta), d F(\mu), d F(\bar{\beta}), J d F(\bar{\mu})\right. \\
& \left.\left.+i \cos \theta_{\mu} d F(\bar{\mu})\right)\right) \\
& +\sum_{\beta, \mu, \rho} \frac{64\left(\cos \theta_{\rho}-\cos \theta_{\mu}\right)}{\sin ^{2} \theta_{\mu} \sin ^{2} \theta_{\rho}}\left|g_{\beta} \mu \rho\right|^{2}
\end{aligned}
$$$$
-\sum_{\beta, \mu, \rho} \frac{64\left(\cos \theta_{\rho}+\cos \theta_{\mu}\right)}{\sin ^{2} \theta_{\mu} \sin ^{2} \theta_{\rho}}\left|g_{\beta} \mu \bar{\rho}\right|^{2}
$$$$
-\sum_{\beta, \mu, \rho} \frac{64\left(\cos \theta_{\rho}+\cos \theta_{\mu}\right)}{\sin ^{2} \theta_{\mu} \sin ^{2} \theta_{\rho}}\left|g_{\beta} \bar{\mu} \rho\right|^{2}
$$$$
+\sum_{\beta, \mu, \rho} \frac{64\left(\cos \theta_{\rho}-\cos \theta_{\mu}\right)}{\sin ^{2} \theta_{\mu} \sin ^{2} \theta_{\rho}}\left|g_{\beta} \overline{\mu \rho}\right|^{2}
$$$$
-\sum_{\beta, \mu, \rho} \frac{64 i\left(\sin ^{2} \theta_{\mu}-\sin ^{2} \theta_{\rho}\right)}{\sin ^{2} \theta_{\mu} \sin ^{2} \theta_{\rho}}\left\langle\nabla_{\bar{\beta}} \bar{\mu}, \rho\right\rangle g_{\beta} \mu \bar{\rho}
$$$$
-\sum_{\beta, \mu, \rho} \frac{64 i\left(\sin ^{2} \theta_{\mu}+\sin ^{2} \theta_{\rho}\right)}{\sin ^{2} \theta_{\mu} \sin ^{2} \theta_{\rho}}\left\langle\nabla_{\bar{\beta}} \mu, \rho\right\rangle g_{\beta} \overline{\mu \rho}
$$$$
+\sum_{\beta, \mu, \rho} \frac{64 i\left(\sin ^{2} \theta_{\mu}+\sin ^{2} \theta_{\rho}\right)}{\sin ^{2} \theta_{\mu} \sin ^{2} \theta_{\rho}}\left\langle\nabla_{\bar{\beta}} \bar{\mu}, \bar{\rho}\right\rangle g_{\beta} \mu \rho
$$$$
+\sum_{\beta, \mu, \rho} \frac{64 i\left(\sin ^{2} \theta_{\mu}-\sin ^{2} \theta_{\rho}\right)}{\sin ^{2} \theta_{\mu} \sin ^{2} \theta_{\rho}}\left\langle\nabla_{\bar{\beta}} \mu, \bar{\rho}\right\rangle g_{\beta} \bar{\mu} \rho
$$ 
(5.33)

$$
\begin{aligned}
& +\sum_{\beta, \mu, \rho} \frac{64 i\left(-\sin ^{2} \theta_{\mu}+\sin ^{2} \theta_{\rho}\right)}{\sin ^{2} \theta_{\mu} \sin ^{2} \theta_{\rho}}\left\langle\nabla_{\beta} \bar{\mu}, \rho\right\rangle g_{\bar{\beta}} \mu \bar{\rho} \\
& +\sum_{\beta, \mu, \rho} \frac{64 i\left(\sin ^{2} \theta_{\mu}-\sin ^{2} \theta_{\rho}\right)}{\sin ^{2} \theta_{\mu} \sin ^{2} \theta_{\rho}}\left\langle\nabla_{\beta} \mu, \bar{\rho}\right\rangle g_{\bar{\beta}} \bar{\mu} \rho \\
& -\sum_{\beta, \mu, \rho} \frac{64\left(\cos \theta_{\mu}-\cos \theta_{\rho}\right)}{\sin ^{2} \theta_{\mu}}\left(\left|\left\langle\nabla_{\beta} \mu, \bar{\rho}\right\rangle\right|^{2}+\left|\left\langle\nabla_{\bar{\beta}} \mu, \bar{\rho}\right\rangle\right|^{2}\right) .
\end{aligned}
$$

By Lemma 5.3,

$$
\begin{aligned}
(5.23)= & \sum_{\beta, \mu, \rho} \frac{64\left(\cos \theta_{\mu}+\cos \theta_{\rho}\right)}{\sin ^{2} \theta_{\mu} \sin ^{2} \theta_{\rho}} \\
& \cdot\left(i g_{\bar{\beta}} \mu \bar{\rho}-i g_{\bar{\beta}} \bar{\rho} \mu-\left(\cos \theta_{\mu}-\cos \theta_{\rho}\right)\left\langle\nabla_{\bar{\beta}} \mu, \bar{\rho}\right\rangle\right) . \\
& \cdot\left(i g_{\beta} \rho \bar{\mu}-i g_{\beta} \bar{\mu} \rho-\left(\cos \theta_{\rho}-\cos \theta_{\mu}\right)\left\langle\nabla_{\beta} \rho, \bar{\mu}\right\rangle\right) \\
=- & \sum_{\beta, \mu, \rho} \frac{64\left(\cos \theta_{\mu}+\cos \theta_{\rho}\right)}{\sin ^{2} \theta_{\mu} \sin ^{2} \theta_{\rho}} g_{\bar{\beta}} \mu \bar{\rho} g_{\beta} \rho \bar{\mu}
\end{aligned}
$$

$$
\begin{aligned}
& +\sum_{\beta, \mu, \rho} \frac{64\left(\cos \theta_{\mu}+\cos \theta_{\rho}\right)}{\sin ^{2} \theta_{\mu} \sin ^{2} \theta_{\rho}}\left|g_{\beta} \bar{\mu} \rho\right|^{2} \\
& +\sum_{\beta, \mu, \rho} \frac{64 i\left(\cos ^{2} \theta_{\mu}-\cos ^{2} \theta_{\rho}\right)}{\sin ^{2} \theta_{\mu} \sin ^{2} \theta_{\rho}} g_{\bar{\beta}} \mu \bar{\rho}\left\langle\nabla_{\beta} \rho, \bar{\mu}\right\rangle \\
& +\sum_{\beta, \mu, \rho} \frac{64\left(\cos \theta_{\mu}+\cos \theta_{\rho}\right)}{\sin ^{2} \theta_{\mu} \sin ^{2} \theta_{\rho}}\left|g_{\beta} \rho \bar{\mu}\right|^{2}
\end{aligned}
$$$$
-\sum_{\beta, \mu, \rho} \frac{64\left(\cos \theta_{\mu}+\cos \theta_{\rho}\right)}{\sin ^{2} \theta_{\mu} \sin ^{2} \theta_{\rho}} g_{\beta} \bar{\mu} \rho g_{\bar{\beta}} \bar{\rho} \mu
$$

$$
-\sum_{\beta, \mu, \rho} \frac{64 i\left(\cos ^{2} \theta_{\mu}-\cos ^{2} \theta_{\rho}\right)}{\sin ^{2} \theta_{\mu} \sin ^{2} \theta_{\rho}}\left\langle\nabla_{\beta} \rho, \bar{\mu}\right\rangle g_{\bar{\beta}} \bar{\rho} \mu
$$

$$
-\sum_{\beta, \mu, \rho} \frac{64 i\left(\cos ^{2} \theta_{\mu}-\cos ^{2} \theta_{\rho}\right)}{\sin ^{2} \theta_{\mu} \sin ^{2} \theta_{\rho}}\left\langle\nabla_{\bar{\beta}} \mu, \bar{\rho}\right\rangle g_{\beta} \rho \bar{\mu}
$$

$$
+\sum_{\beta, \mu, \rho} \frac{64 i\left(\cos ^{2} \theta_{\mu}-\cos ^{2} \theta_{\rho}\right)}{\sin ^{2} \theta_{\mu} \sin ^{2} \theta_{\rho}}\left\langle\nabla_{\bar{\beta}} \mu, \bar{\rho}\right\rangle g_{\beta} \bar{\mu} \rho
$$

$$
+\sum_{\beta, \mu, \rho} \frac{64\left(\cos ^{2} \theta_{\mu}-\cos ^{2} \theta_{\rho}\right)}{\sin ^{2} \theta_{\mu} \sin ^{2} \theta_{\rho}}\left(\cos \theta_{\rho}-\cos \theta_{\mu}\right)\left\langle\nabla_{\bar{\beta}} \mu, \bar{\rho}\right\rangle\left\langle\nabla_{\beta} \rho, \bar{\mu}\right\rangle .
$$

Immediately we have, $(5.27)+(5.36)=(5.32)+(5.41)=(5.33)+(5.37)=$ 0 , and interchanging $\mu$ with $\rho$ in (5.26), (5.34) and in (5.40), we get, (5.26)+ 
$(5.38)=(5.29)+(5.40)=(5.34)+(5.39)=0$. Note that

$$
\sum_{\mu, \rho} \frac{\left(\cos \theta_{\mu}-\cos \theta_{\rho}\right)}{\sin ^{2} \theta_{\mu}}\left|\left\langle\nabla_{\beta} \mu, \bar{\rho}\right\rangle\right|^{2}=\sum_{\mu, \rho} \frac{\left(\cos \theta_{\rho}-\cos \theta_{\mu}\right)}{\sin ^{2} \theta_{\rho}}\left|\left\langle\nabla_{\bar{\beta}} \mu, \bar{\rho}\right\rangle\right|^{2} .
$$

Hence $(5.35)+(5.42)=0$. Then,

$2 \triangle \kappa$

$$
\begin{aligned}
& =\sum_{\beta, \mu} \frac{32 i}{\sin ^{2} \theta_{\mu}} R^{N}\left(d F(\beta), d F(\bar{\beta}), d F(\mu), J d F(\bar{\mu})+i \cos \theta_{\mu} d F(\bar{\mu})\right) \\
& +\sum_{\beta, \mu} \frac{64}{\sin ^{2} \theta_{\mu}} \operatorname{Im}\left(R^{N}(d F(\beta), d F(\mu), d F(\bar{\beta}), J d F(\bar{\mu})\right. \\
& \left.\left.\quad+i \cos \theta_{\mu} d F(\bar{\mu})\right)\right)
\end{aligned}
$$

$$
\begin{aligned}
& +\sum_{\beta, \mu, \rho}-\frac{64\left(\cos \theta_{\mu}+\cos \theta_{\rho}\right)}{\sin ^{2} \theta_{\mu} \sin ^{2} \theta_{\rho}}\left(g_{\bar{\beta}} \mu \bar{\rho} g_{\beta} \rho \bar{\mu}+g_{\beta} \bar{\mu} \rho g_{\bar{\beta}} \bar{\rho} \mu\right) \\
& +\sum_{\beta, \mu, \rho} \frac{64\left(\cos \theta_{\rho}-\cos \theta_{\mu}\right)}{\sin ^{2} \theta_{\mu} \sin ^{2} \theta_{\rho}}\left(\left|g_{\beta} \mu \rho\right|^{2}+\left|g_{\bar{\beta}} \mu \rho\right|^{2}\right) \\
& -\sum_{\beta, \mu, \rho} \frac{64 i\left(\sin ^{2} \theta_{\mu}+\sin ^{2} \theta_{\rho}\right)}{\sin ^{2} \theta_{\mu} \sin ^{2} \theta_{\rho}}\left\langle\nabla_{\bar{\beta}} \mu, \rho\right\rangle g_{\beta} \overline{\mu \rho}
\end{aligned}
$$$$
+\sum_{\beta, \mu, \rho} \frac{64 i\left(\sin ^{2} \theta_{\mu}+\sin ^{2} \theta_{\rho}\right)}{\sin ^{2} \theta_{\mu} \sin ^{2} \theta_{\rho}}\left\langle\nabla_{\bar{\beta}} \bar{\mu}, \bar{\rho}\right\rangle g_{\beta} \mu \rho .
$$

Using Lemma 5.3, and interchanging $\rho$ by $\mu$ when necessary,

$$
\begin{aligned}
&(5.45)+(5.46) \\
&=\sum_{\beta, \mu, \rho}-\frac{64 i}{\sin ^{2} \theta_{\rho}}\left\langle\nabla_{\bar{\beta}} \mu, \rho\right\rangle g_{\beta} \overline{\mu \rho}-\frac{64 i}{\sin ^{2} \theta_{\mu}}\left\langle\nabla_{\bar{\beta}} \mu, \rho\right\rangle g_{\beta} \overline{\mu \rho} \\
&+\frac{64 i}{\sin ^{2} \theta_{\mu}}\left\langle\nabla_{\bar{\beta}} \bar{\mu}, \bar{\rho}\right\rangle g_{\beta} \mu \rho+\frac{64 i}{\sin ^{2} \theta_{\rho}}\left\langle\nabla_{\bar{\beta}} \bar{\mu}, \bar{\rho}\right\rangle g_{\beta} \mu \rho \\
&= \sum_{\beta, \mu, \rho} \frac{-64 i}{\sin ^{2} \theta_{\mu}}\left\langle\nabla_{\bar{\beta}} \mu, \rho\right\rangle\left(g_{\beta} \overline{\mu \rho}-g_{\beta} \overline{\rho \mu}\right) \\
&+\sum_{\beta, \mu, \rho} \frac{64 i}{\sin ^{2} \theta_{\mu}}\left\langle\nabla_{\bar{\beta}} \bar{\mu}, \bar{\rho}\right\rangle\left(g_{\beta} \mu \rho-g_{\beta} \rho \mu\right) \\
&= \sum_{\beta, \mu, \rho} \frac{64}{\sin ^{2} \theta_{\mu}}\left\langle\nabla_{\bar{\beta}} \mu, \rho\right\rangle\left(\cos \theta_{\mu}+\cos \theta_{\rho}\right)\left\langle\nabla_{\beta} \bar{\mu}, \bar{\rho}\right\rangle \\
&+\frac{64}{\sin ^{2} \theta_{\mu}}\left\langle\nabla_{\bar{\beta}} \bar{\mu}, \bar{\rho}\right\rangle\left(\cos \theta_{\mu}+\cos \theta_{\rho}\right)\left\langle\nabla_{\beta} \mu, \rho\right\rangle
\end{aligned}
$$




$$
=\sum_{\beta, \mu, \rho} \frac{64\left(\cos \theta_{\mu}+\cos \theta_{\rho}\right)}{\sin ^{2} \theta_{\mu}}\left(\left|\left\langle\nabla_{\beta} \mu, \rho\right\rangle\right|^{2}+\left|\left\langle\nabla_{\beta} \mu, \rho\right\rangle\right|^{2}\right) .
$$

Obviously

$$
(5.44)=\sum_{\beta, \mu, \rho} \frac{-128\left(\cos \theta_{\mu}+\cos \theta_{\rho}\right)}{\sin ^{2} \theta_{\mu} \sin ^{2} \theta_{\rho}} \operatorname{Re}\left(g_{\beta} \mu \bar{\rho} g_{\bar{\beta}} \rho \bar{\mu}\right) .
$$

From (1.4), (2.1), and the $J$-invariance of Ricci,

$$
(5.43)=8 i \sum_{\beta} \operatorname{Ricci}^{N}(J d F(\beta), d F(\bar{\beta})),
$$

and the expression of the Proposition follows.

After completion and posting of this work in the e-print archive (with no. math.DG/0002050) my attention was drawn to a related paper by A.Ghigi $[\mathbf{G}]$, published in the meantime, which contains the same result as ours for the case $R \neq 0$ and $n=2$, but proved in a different way.

Acknowledgments. We would like to thank very much Professor James Eells and Professor Claude LeBrun for helpful discussions and encouragement.

\section{References}

[B] M. Berger, Sur les groupes d'holonomie des variétés à connexion affine et des variétés riemannienes, Bull. Soc. Math. France, 83 (1955), 279-330, MR 18,149a, Zbl 0068.36002.

[Ch-W] S.S. Chern and J.G. Wolfson, Minimal surfaces by moving frames, Amer. J. Math., 105 (1983), 59-83, MR 84i:53056, Zbl 0521.53050.

[E-L] J. Eells and L. Lemaire, Selected topics in harmonic maps, C.B.M.S. Regional Conf. Series, 50, A.M.S. (1983), MR 85g:58030, Zbl 0515.58011.

[G] A. Ghigi, A generalization of Cayley submanifolds, IMRN, 15 (2000), 787-800, MR 2001i:53077, Zbl 0963.53027.

[Mi-Mo] M.J. Micallef and J.D. Moore, Minimal two-spheres and the topology of manifolds with positive curvature on totally isotropic two-planes, Annals of Math., 127 (1988), 199-227, MR 89e:53088, Zbl 0661.53027.

[O] Y. Ohnita, Minimal surfaces with constant curvature and Kähler angle in complex space forms, Tsukuba J. Math., 13(1) (1989), 191-207, MR 90c:53157, Zbl 0678.53055.

[O-V] Y. Ohnita and G. Valli, Pluriharmonic maps into compact Lie groups and factorization into unitons, Proc. London Math. Soc., 61 (1990), 546-570, MR 91i:58034, Zbl 0677.58019.

[S-V] I. Salavessa and G. Valli, Broadly-pluriminimal submanifolds of Kähler-Einstein manifolds, Yokohama Math. J., 8(2) (2001), 181-199, MR 2002f:53110. 
[W] J.G. Wolfson, Minimal surfaces in Kähler surfaces and Ricci curvature, J. Differential Geom., 29 (1989), 281-294, MR 90d:53073, Zbl 0667.53044.

Received April 10, 2000 and revised March 9, 2001. The second author passed away on October 2, 1999.

Centro de Física das Interacções Fundamentais

Instituto Superior TÉCNICO

Edifício Cî̂nCIA, Piso 3

Av. Rovisco Pais

P-1049-001 LisBoA

Portugal

E-mail address: isabel@cartan.ist.utl.pt

2 Dipartimento di Matematica

Università Di PAVIA

Via AbBiategrasso 215

27100 PAVIA

ITALY 